\title{
On the dynamics of particle sedimentation in viscoelastic fluids: A numerical study on particle chaining in two-dimensional narrow channels
}

\author{
Tsorng-Whay Pan \\ Department of Mathematics, University of Houston, Houston, Texas 77204, USA \\ Roland Glowinski \\ Department of Mathematics, University of Houston, Houston, Texas 77204, USA \\ Department of Mathematics, Hong Kong Baptist University, Hong Kong
}

\begin{abstract}
The main goal of this article is to generalize to two-dimensional particulate viscoelastic channel flow of the FENE-CR type, the distributed Lagrange multiplier based fictitious domain (FD/DLM) methodology the authors employed in [J. Non-Newtonian Fluid Mech. 156 (2009) 95] for the simulation of viscoelastic particulate flow of the Oldroyd-B type. As in the above reference the methodology we employ here is based on a FD/DLM technique, combined with operator-splitting, a Cholesky factorization treatment (à la Lozinski-Owens) of the conformation tensor, and with appropriate finite element approximations of the various functions contained in the flow model. In this article, $N$ being the number of particles and $L$ the fluid polymer extension limit, we have used the methodology briefly sketched above to investigate, both in the transient and time asymptotic regimes, the influence of $N, L$, and of the relaxation time, on the formation of vertical chains of particles and on the maximal number of particles these chains do contain. We have verified in particular that small values of $L$ do not authorize long particle chains, and also that one recovers, as expected, Oldroyd-B from FENE-CR as $L \rightarrow+\infty$.
\end{abstract}

Keywords: FENE-CR viscoelastic fluids; Oldroyd-B viscoelastic fluids; operator-splitting; fictitious domains; particle chaining; conformation tensor; positive definiteness.

\section{Introduction}

The motion of particles in non-Newtonian fluids is not only of fundamental theoretical interest, but is also of importance in many applications to industrial processes involving particle-laden materials (see, e.g., [1] and [2]). For example, during the hydraulic fracturing operations used in oil and gas wells, suspensions of solid particles in polymeric solutions are pumped into hydraulically-induced fractures. The particles must prop these channels open to enhance the rate of oil recovery [3]. During the shut-in stage, proppant settling is pronounced when the fluid pressure decreases due to the end of the hydraulic fracturing process. The study of particle chain during settling in vertical channel can help practitioners to understand better the mechanism of proppant agglomeration in narrow fracture zones [4].

Although numerical methods for the simulation of particles moving in Newtonian fluids have been very successful, the situation is much more complicated and challenging if the fluid is viscoelastic. One of the difficulties (e.g., see [5], [6]) one encounters when attempting the simulation of viscoelastic flow is the breakdown of the numerical method. Indeed, most experts agree that one can expect the breakdown of the numerical method if the time-discretization one employs does not preserve during the entire time integration the

*pan@math.uh.edu, roland@math.uh.edu 
positive definiteness of the discrete conformation tensor. To preserve this positive definiteness property of the conformation tensor, several approaches have been advocated (e.g., [7], [8], [9] and [10]). In this article, we have followed the quite elegant approach introduced by Lozinski and Owens [10]: the Lozinski-Owens approach relies on the Cholesky factorization $\mathbf{C}=\mathbf{A} \mathbf{A}^{T}$ of the conformation tensor, and then on a reformulation in terms of $\mathbf{A}$ of the time dependent equation modeling the evolution of $\mathbf{C}$, providing automatically that $\mathbf{C}$ is at least positive semi-definite (and symmetric). The Cholesky factorization based method introduced in [10] has been applied in [11], via an operator splitting time discretization scheme coupled to a FD/DLM method, to the simulation of particulate flows in Oldroyd-B fluids. It is worth and important to mention that the Oldroyd-B related numerical experiments reported in [11] strongly suggest that the computed solutions converge to a limit as the space and time discretization steps get small enough. Generalizing the methodology developed in [11] to viscoelastic fluids of the FENE-CR is straightforward, our motivation at doing so being that, as advocated in [12], FENE-CR is a more realistic model than Oldroyd-B. What we have in mind in particular is to compare the chain formation phenomena taking place during the settling of two, three, and six disks in two-dimensional vertical narrow channels containing either Oldroyd-B or FENE-CR fluids (the Oldroyd-B cases have been already considered in [11] and show particle chain formation). In the FENE-CR case, we expect that for large values of $L$, FENE-CR will behave like Oldroyd-B, a prediction confirmed by our numerical experiments. Actually, our numerical experiments show also that for the two and three disk cases, we still have chain formation with the smaller values of $L$ we considered, however, if one increases the relaxation time, the transient regimes associated with these small values of $L$ are quite different from those associated with large values or with Oldroy-B, even if asymptotically one obtains similar chain configurations. For six disks settling in a FENE-CR viscoelastic fluid, depending of the value of $L$ the formation of four to six disk chains is observed. For the smallest values of $L$, our simulations show that a FENE-CR fluid cannot sustain chains of more than three particles, and indeed, two chains of three particles each are formed. Similar results are observed for ten disks settling in the same type of two-dimensional channels. Further numerical experiments allow us to conclude that the smaller $L$, the shorter the particle chains and the smaller the size of the particle clusters. This article is organized as follows: In Section 2 we present a model for FENE-CR particulate flow and its equivalent distributed Lagrange multiplier based fictitious domain (FD/DLM) formulation. Then we discuss the space-time discretization of the resulting systems of equations, based on a methodology combining operator-splitting and finite element approximations, a particular attention being given to the Cholesky factorization based on Lozinski-Owens treatment of the conformation tensor. The results of numerical experiments are presented in Section 3, which includes also a detailed discussion of the influence of the polymer extension coefficient $L$ on the number of particles present in the vertical chains.

\section{Mathematical formulations and numerical methods}

\subsection{Governing equations and their related FD/DLM formulation}

Following [11], we will describe first the models and computational methodologies to be employed in this article, including a discussion of the Lozinski-Owens factorization approach. Let $\Omega$ be a bounded twodimensional (2D) domain and let $\Gamma$ be its boundary. We suppose that $\Omega$ is filled with a viscoelastic fluid of either Oldroyd-B or FENE-CR type of density $\rho_{f}$ and that it contains $N$ moving rigid particles of density $\rho_{s}$ (see Figure 1). Let $B(t)=\cup_{i=1}^{N} B_{i}(t)$ where $B_{i}(t)$ is the $i$ th rigid particle in the fluid for $i=1, \ldots, N$. We denote by $\partial B_{i}(t)$ the boundary of $B_{i}(t)$. For some $T>0$, the governing equations for the fluid-particle 


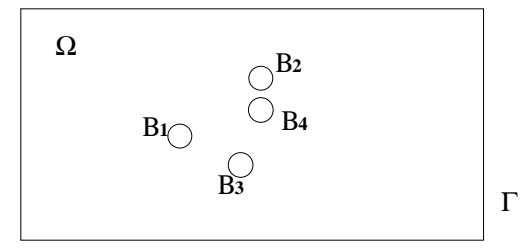

Figure 1: An example of a two-dimensional flow region with four circular particles.

system are

$$
\begin{aligned}
& \rho_{f}\left(\frac{\partial \mathbf{u}}{\partial t}+(\mathbf{u} \cdot \nabla) \mathbf{u}\right)=\rho_{f} \mathbf{g}-\nabla p+2 \mu \nabla \cdot \mathbf{D}(\mathbf{u})+\nabla \cdot \boldsymbol{\sigma}^{p} \quad \text { in } \Omega \backslash \overline{B(t)}, t \in(0, T), \\
& \nabla \cdot \mathbf{u}=0 \quad \text { in } \Omega \backslash \overline{B(t)}, t \in(0, T), \\
& \mathbf{u}(\mathbf{x}, 0)=\mathbf{u}_{0}(\mathbf{x}), \quad \forall \mathbf{x} \in \Omega \backslash \overline{B(0)} \text {, with } \nabla \cdot \mathbf{u}_{0}=0, \\
& \mathbf{u}=\mathbf{g}_{0} \quad \text { on } \Gamma \times(0, T), \text { with } \int_{\Gamma} \mathbf{g}_{0} \cdot \mathbf{n} d \Gamma=0, \\
& \mathbf{u}=\mathbf{V}_{p, i}+\omega_{i} \overrightarrow{\mathbf{G}_{i} \mathbf{x}}, \forall \mathbf{x} \in \partial B_{i}(t), i=1, \cdots, N, \\
& \frac{\partial \mathbf{C}}{\partial t}+(\mathbf{u} \cdot \boldsymbol{\nabla}) \mathbf{C}-(\nabla \mathbf{u}) \mathbf{C}-\mathbf{C}(\nabla \mathbf{u})^{t}=-\frac{f(\mathbf{C})}{\lambda_{1}}(\mathbf{C}-\mathbf{I}) \text { in } \Omega \backslash \overline{B(t)}, t \in(0, T), \\
& \mathbf{C}(\mathbf{x}, 0)=\mathbf{C}_{0}(\mathbf{x}), \mathbf{x} \in \Omega \backslash \overline{B(0)}, \\
& \mathbf{C}=\mathbf{C}_{L}, \quad \text { on } \Gamma^{-}(t),
\end{aligned}
$$

where $\mathbf{u}$ is the flow velocity, $p$ is the pressure, $\mathbf{g}$ denotes gravity, $\mu=\eta_{1} \lambda_{2} / \lambda_{1}$ is the solvent viscosity of the fluid, $\eta=\eta_{1}-\mu$ is the elastic viscosity of the fluid, $\eta_{1}$ is the fluid viscosity, $\lambda_{1}$ is the relaxation time of the fluid, $\lambda_{2}$ is the retardation time of the fluid, $\mathbf{n}$ is the outer normal unit vector at $\Gamma, \Gamma^{-}(t)$ is the upstream portion of $\Gamma$ at time $t$. The polymeric stress tensor $\boldsymbol{\sigma}^{p}$ in $(1)$ is given by $\boldsymbol{\sigma}^{p}=\frac{\eta}{\lambda_{1}} f(\mathbf{C})(\mathbf{C}-\mathbf{I})$, where the conformation tensor $\mathbf{C}$ is symmetric and positive definite (see [13]) and $\mathbf{I}$ is the identity tensor. Setting $f=1$ corresponds to the Oldroyd-B model while

$$
f(\mathbf{C})=\frac{L^{2}}{L^{2}-\operatorname{tr}(\mathbf{C})}
$$

corresponds to the FENE-CR model [14], where $\operatorname{tr}(\mathbf{C})$ is the trace of the conformation tensor $\mathbf{C}$ and the quantity $L$ is the maximum extension of the immersed polymer coils and referred as the extensibility of the immersed polymer coils. The Oldroyd-B model then is a special case associated with infinite extensibility.

In (5), the no-slip condition holds on the boundary of the $i$ th particle, $\mathbf{V}_{p, i}$ is its translation velocity, $\omega_{i}$ is its angular velocity, $\mathbf{G}_{i}=\left\{G_{i, 1}, G_{i, 2}\right\}^{t}$ is the center of mass, and finally ${\overrightarrow{\mathbf{G}_{i} \mathbf{x}}}^{\perp}$ is $\left\{-\left(x_{2}-G_{i, 2}\right),\left(x_{1}-G_{i, 1}\right)\right\}^{t}$ for the rotation with respect to the mass center $\mathbf{G}_{i}$ (for the $2 \mathrm{D}$ cases considered in this article). The motion of the particles is modeled by Newton's laws:

$$
\begin{aligned}
& M_{p, i} \frac{d \mathbf{V}_{p, i}}{d t}=M_{p, i} \mathbf{g}+\mathbf{F}_{i}+\mathbf{F}_{i}^{r}, \\
& I_{p, i} \frac{d \omega_{i}}{d t}=F_{i}^{t}, \\
& \frac{d \mathbf{G}_{i}}{d t}=\mathbf{V}_{p, i}, \\
& \mathbf{G}_{i}(0)=\mathbf{G}_{i}^{0}, \mathbf{V}_{p, i}(0)=\mathbf{V}_{p, i}^{0}, \omega_{i}(0)=\omega_{i}^{0},
\end{aligned}
$$


for $i=1, \ldots, N$, where in (10)-(13), $M_{p, i}$ and $I_{p, i}$ are the the mass and the inertia of the $i$ th particle, respectively, $\mathbf{F}_{i}^{r}$ is a short range repulsion force imposed on the $i$ th particle by other particles and the wall to prevent particle/particle and particle/wall penetration (see [15] for details), and $\mathbf{F}_{i}$ and $F_{i}^{t}$ denote the hydrodynamic force and the associated torque imposed on the $i$ th particle by the fluid, respectively.

To avoid frequent remeshing and the difficulties associated with mesh generation for a time-varying domain in which the rigid particles can be very close to each other, especially for three dimensional particulate flow, we have extended the governing equations to the entire domain $\Omega$ (the fictitious domain here). For the fictitious-domain-based variational formulation of the governing equations, we consider for simplicity only one rigid particle $B(t)$ (a disk in 2D) in the fluid domain, without loss of generality. Let us define first the following functional spaces

$$
\begin{aligned}
& \mathbf{V}_{\mathbf{g}_{0}(t)}=\left\{\mathbf{v} \mid \mathbf{v} \in\left(H^{1}(\Omega)\right)^{2}, \mathbf{v}=\mathbf{g}_{0}(t) \text { on } \Gamma\right\} \\
& L_{0}^{2}(\Omega)=\left\{q \mid q \in L^{2}(\Omega), \int_{\Omega} q d \mathbf{x}=0\right\} \\
& \mathbf{V}_{\mathbf{C}_{L}(t)}=\left\{\mathbf{C} \mid \mathbf{C} \in\left(H^{1}(\Omega)\right)^{2 \times 2}, \mathbf{C}=\mathbf{C}_{L}(t) \text { on } \Gamma^{-}(t)\right\} \\
& \mathbf{V}_{\mathbf{C}_{0}(t)}=\left\{\mathbf{C} \mid \mathbf{C} \in\left(H^{1}(\Omega)\right)^{2 \times 2}, \mathbf{C}=0 \text { on } \Gamma^{-}(t)\right\} \\
& \Lambda(t)=\left(H^{1}(B(t))\right)^{2} .
\end{aligned}
$$

To derive the FD/DLM model, one takes advantage of several facts: (i) if $\mathbf{v}$ is a rigid body motion velocity field of $B(t), \mathbf{D}(\mathbf{v})=\mathbf{0}$, implying $\boldsymbol{\nabla} \cdot \mathbf{v}=0$. (ii) $\mathbf{C}=\mathbf{I}$ in $B(t)$. Following the methodologies developed in $[11,15,16,17]$, a fictitious domain formulation of the governing equations (1)-(13) reads as follows:

For a.e. $t>0$, find $\mathbf{u}(t) \in \mathbf{V}_{\mathbf{g}_{0}(t)}, p(t) \in L_{0}^{2}(\Omega), \mathbf{C}(t) \in \mathbf{V}_{\mathbf{C}_{L}(t)}, \mathbf{V}(t) \in \mathbb{R}^{2}, \mathbf{G}(t) \in \mathbb{R}^{2}, \omega(t) \in \mathbb{R}$, $\boldsymbol{\lambda}(t) \in \Lambda(t)$ such that

$$
\begin{aligned}
& \rho_{f} \int_{\Omega}\left[\frac{\partial \mathbf{u}}{\partial t}+(\mathbf{u} \cdot \boldsymbol{\nabla}) \mathbf{u}\right] \cdot \mathbf{v} d \mathbf{x}+2 \mu \int_{\Omega} \mathbf{D}(\mathbf{u}): \mathbf{D}(\mathbf{v}) d \mathbf{x}-\int_{\Omega} p \boldsymbol{\nabla} \cdot \mathbf{v} d \mathbf{x} \\
& \left\{-\int_{\Omega} \mathbf{v} \cdot\left(\boldsymbol{\nabla} \cdot \boldsymbol{\sigma}^{p}\right) d \mathbf{x}+\left(1-\rho_{f} / \rho_{s}\right)\left\{M_{p} \frac{d \mathbf{V}}{d t} \cdot \mathbf{Y}+I_{p} \frac{d \omega}{d t} \theta\right\}\right. \\
& \left\{-<\boldsymbol{\lambda}, \mathbf{v}-\mathbf{Y}-\theta \overrightarrow{\mathbf{G x}}{ }^{\perp}>_{B(t)}-\mathbf{F}^{r} \cdot \mathbf{Y}\right. \\
& \begin{array}{l}
=\rho_{f} \int_{\Omega} \mathbf{g} \cdot \mathbf{v} d \mathbf{x}+\left(1-\rho_{f} / \rho_{s}\right) M_{p} \mathbf{g} \cdot \mathbf{Y}, \\
\forall\{\mathbf{v}, \mathbf{Y}, \theta\} \in\left(H_{0}^{1}(\Omega)\right)^{2} \times \mathbb{R}^{2} \times \mathbb{R}
\end{array} \\
& \int_{\Omega} q \nabla \cdot \mathbf{u}(t) d \mathbf{x}=0, \forall q \in L^{2}(\Omega), \\
& <\boldsymbol{\eta}, \mathbf{u}(\mathbf{x}, t)-\mathbf{V}(t)-\omega(t) \overrightarrow{\mathbf{G}(t) \mathbf{x}}^{\perp}>_{B(t)}=0, \quad \forall \boldsymbol{\eta} \in \boldsymbol{\Lambda}(t), \\
& \int_{\Omega}\left(\frac{\partial \mathbf{C}}{\partial t}+(\mathbf{u} \cdot \boldsymbol{\nabla}) \mathbf{C}-(\boldsymbol{\nabla} \mathbf{u}) \mathbf{C}-\mathbf{C}(\boldsymbol{\nabla} \mathbf{u})^{t}\right): \mathbf{s} d \mathbf{x} \\
& =-\int_{\Omega} \frac{f(\mathbf{C})}{\lambda_{1}}(\mathbf{C}-\mathbf{I}): \mathbf{s} d \mathbf{x}, \forall \mathbf{s} \in \mathbf{V}_{\mathbf{C}_{0}(t)} \text {, with } \mathbf{C}=\mathbf{I} \text { in } B(t) \text {, } \\
& \frac{d \mathbf{G}}{d t}=\mathbf{V} \text {, } \\
& \mathbf{C}(\mathbf{x}, 0)=\mathbf{C}_{0}(\mathbf{x}), \forall \mathbf{x} \in \Omega \text {, with } \mathbf{C}_{0}=\mathbf{I} \text { in } B(0) \text {, } \\
& \mathbf{G}(0)=\mathbf{G}_{0}, \mathbf{V}(0)=\mathbf{V}_{0}, \omega(0)=\omega_{0}, B(0)=B_{0}, \\
& \mathbf{u}(\mathbf{x}, 0)=\left\{\begin{array}{l}
\mathbf{u}_{0}(\mathbf{x}), \forall \mathbf{x} \in \Omega \backslash \overline{B_{0}}, \\
\mathbf{V}_{0}+\omega_{0} \overline{\mathbf{G}_{0} \mathbf{x}}, \forall \mathbf{x} \in \overline{B_{0}} .
\end{array}\right.
\end{aligned}
$$


In (14) the Lagrange multiplier $\boldsymbol{\lambda}$ defined over $B$ can be viewed as an extra body force maintaining the rigid body motion inside $B$. The conformation tensor $\mathbf{C}$ inside the rigid particle is extended as the identity tensor $\mathbf{I}$ as in (17) since the polymeric stress tensor is zero inside the rigid particle. In equation (14), since $\mathbf{u}$ is divergence free and $\mathbf{v}$ vanishes on $\Gamma$, we have $2 \int_{\Omega} \mathbf{D}(\mathbf{u}): \mathbf{D}(\mathbf{v}) d \mathbf{x}=\int_{\Omega} \boldsymbol{\nabla} \mathbf{u}: \nabla \mathbf{v} d \mathbf{x}, \forall \mathbf{v} \in\left(H_{0}^{1}(\Omega)\right)^{2}$. This is a substantial simplification from the computational point of view, which is another advantage of the fictitious domain approach. With this simplification, we can use, as shown in the following section, fast solvers for the elliptic problems in order to speed up computations. Also the gravity term $\mathbf{g}$ in (14) can be absorbed in the pressure term.

\subsection{Finite element approximation}

In order to solve problem (14)-(21) numerically, we shall discretize $\Omega$ using an uniform finite element mesh $\mathcal{T}_{h}$ for the velocity and conformation tensor, where $h$ is the mesh size, and a twice coarser uniform mesh $\mathcal{T}_{2 h}$ for the pressure. The following finite dimensional spaces are employed for approximating $\mathbf{V}_{\mathbf{g}_{0}(t)}$, $\left(H_{0}^{1}(\Omega)\right)^{2}, L^{2}(\Omega), L_{0}^{2}(\Omega), \mathbf{V}_{\mathbf{C}_{L}(t)}, \mathbf{V}_{\mathbf{C}_{0}}$, respectively,

$$
\begin{aligned}
& \mathbf{V}_{\mathbf{g}_{0 h}(t)}=\left\{\mathbf{v}_{h}\left|\mathbf{v}_{h} \in\left(C^{0}(\bar{\Omega})\right)^{2}, \mathbf{v}_{h}\right|_{E} \in\left(P_{1}\right)^{2}, \forall E \in \mathcal{T}_{h},\left.\mathbf{v}_{h}\right|_{\Gamma}=\mathbf{g}_{0 h}(t)\right\}, \\
& \mathbf{V}_{0 h}=\left\{\mathbf{v}_{h}\left|\mathbf{v}_{h} \in\left(C^{0}(\bar{\Omega})\right)^{2}, \mathbf{v}_{h}\right|_{E} \in\left(P_{1}\right)^{2}, \forall E \in \mathcal{T}_{h},\left.\mathbf{v}_{h}\right|_{\Gamma}=0\right\}, \\
& L_{h}^{2}=\left\{q_{h}\left|q_{h} \in C^{0}(\bar{\Omega}), q_{h}\right|_{E} \in P_{1}, \forall E \in \mathcal{T}_{2 h}\right\} \\
& L_{0 h}^{2}=\left\{q_{h} \mid q_{h} \in L_{h}^{2}, \int_{\Omega} q_{h} d \mathbf{x}=\mathbf{0}\right\}, \\
& \mathbf{V}_{\mathbf{C}_{L h}(t)}=\left\{\mathbf{s}_{h}\left|\mathbf{s}_{h} \in\left(C^{0}(\bar{\Omega})\right)^{2 \times 2}, \mathbf{s}_{h}\right|_{E} \in\left(P_{1}\right)^{2 \times 2}, \forall E \in \mathcal{T}_{h},\left.\mathbf{s}_{h}\right|_{\Gamma_{h}^{-}(t)}=\mathbf{C}_{L h}(t)\right\}, \\
& \mathbf{V}_{\mathbf{C}_{0 h}(t)}=\left\{\mathbf{s}_{h}\left|\mathbf{s}_{h} \in\left(C^{0}(\bar{\Omega})\right)^{2 \times 2}, \mathbf{s}_{h}\right|_{E} \in\left(P_{1}\right)^{2 \times 2}, \forall E \in \mathcal{T}_{h},\left.\mathbf{s}_{h}\right|_{\Gamma_{h}^{-}(t)}=0\right\}
\end{aligned}
$$

where $P_{1}$ is the space of the polynomials in two variables of degree $\leq 1, \mathbf{g}_{0 h}(t)$ is an approximation of $\mathbf{g}_{0}$ satisfying $\int_{\Gamma} \mathbf{g}_{0 h}(t) \cdot \mathbf{n} d \Gamma=0$, and $\Gamma_{h}^{-}(t)=\left\{\mathbf{x} \mid \mathbf{x} \in \Gamma, \mathbf{g}_{0 h}(\mathbf{x}, t) \cdot \mathbf{n}(\mathbf{x})<0\right\}$. The discrete Lagrange multiplier space $\Lambda_{h}(t)$ is defined as follows: let $\left\{\mathbf{x}_{i}\right\}_{i=1}^{K}$ be a set of points from $\overline{B(t)}$ that covers $\overline{B(t)}$ evenly; then we define

$$
\boldsymbol{\Lambda}_{h}(t)=\left\{\boldsymbol{\eta} \mid \boldsymbol{\eta}=\sum_{j=1}^{K} \boldsymbol{\eta}_{j} \delta\left(\mathbf{x}-\mathbf{x}_{j}\right), \boldsymbol{\eta}_{j} \in \mathbb{R}^{2}, \forall j=1, \ldots, K\right\},
$$

where $\mathbf{x} \rightarrow \delta\left(\mathbf{x}-\mathbf{x}_{j}\right)$ is the Dirac measure at $\mathbf{x}_{j}$. Then instead of the inner product of $\left(H^{1}\left(B_{h}(t)\right)^{2}\right.$, we shall use the bilinear functional $\langle\cdot, \cdot\rangle_{B_{h}(t)}$ defined by

$$
<\boldsymbol{\eta}, \mathbf{v}>_{B_{h}(t)}=\sum_{j=1}^{K} \boldsymbol{\eta}_{j} \cdot \mathbf{v}\left(\mathbf{x}_{j}\right), \forall \boldsymbol{\eta} \in \boldsymbol{\Lambda}_{h}(t), \mathbf{v} \in \mathbf{V}_{\mathbf{g}_{0 h}(t)} \text { or } \mathbf{V}_{0 h}
$$

Using the above pairing implies that the rigid body motion of $B(t)$ is forced via a collocation method [15].

Then a discrete analogue of problem (14)-(21) can be obtained using the above finite dimensional spaces.

\subsection{An operator splitting time-discretization scheme}

Consider the following initial value problem:

$$
\frac{d \phi}{d t}+A(\phi)=0 \text { on }(0, T), \quad \phi(0)=\phi_{0}
$$

with $0<T \leq+\infty$. We suppose that operator $A$ has a decomposition such as $A=\sum_{j=1}^{J} A_{j}$ with $J \geq 2$. Let $\tau(>0)$ be a time-discretization step, we denote $n \tau$ by $t^{n}$. With $\phi^{n}$ denoting an approximation of $\phi\left(t^{n}\right)$, the Lie scheme [18], [20] and [21] reads as follows: 
For $n \geq 0$, assuming that $\phi^{n}$ is known (with $\phi^{0}=\phi_{0}$ ), compute $\phi^{n+1}$ via

$$
\left\{\begin{array}{l}
\frac{d \phi}{d t}+A_{j}(\phi)=0 \text { on }\left(t^{n}, t^{n+1}\right) \\
\phi\left(t^{n}\right)=\phi^{n+(j-1) / J} ; \phi^{n+j / J}=\phi\left(t^{n+1}\right),
\end{array}\right.
$$

for $j=1, \ldots, J$. The Lie scheme is first order accurate, but its low order of accuracy is compensated by its simplicity, making it (relatively) easy to implement, and by its robustness. Some classical operator splitting techniques with application to the Navier-Stokes equations have been discussed in [19], [20] and [21] in details.

The Lie operator splitting scheme allows us to decouple the following difficulties:

(1) The incompressibility condition, and the related unknown pressure.

(2) The advection terms.

(3) The rigid-body motion in $B_{h}(t)$, and the related Lagrange multiplier $\boldsymbol{\lambda}_{h}$.

The constitutive equation satisfied by the conformation tensor $\mathbf{C}$ is split with $J=3$ in order to show how the Lozinski-Owens factorization approach works out. Suppose that $\mathbf{C}^{n}$ and $\mathbf{u}$ are known, we solve

$$
\begin{aligned}
& \left\{\begin{array}{l}
\frac{\partial \mathbf{C}}{\partial t}+(\mathbf{u} \cdot \boldsymbol{\nabla}) \mathbf{C}=\mathbf{0} \text { in } \Omega \times\left(t^{n}, t^{n+1}\right), \\
\mathbf{C}\left(t^{n}\right)=\mathbf{C}^{n} ; \mathbf{C}^{n+1 / 3}=\mathbf{C}\left(t^{n+1}\right),
\end{array}\right. \\
& \left\{\begin{array}{l}
\frac{\partial \mathbf{C}}{\partial t}-(\boldsymbol{\nabla} \mathbf{u}) \mathbf{C}-\mathbf{C}(\boldsymbol{\nabla} \mathbf{u})^{t}+\frac{f\left(\mathbf{C}^{n+1 / 3}\right)}{\lambda_{1}} \mathbf{C}=\mathbf{0} \text { in } \Omega \times\left(t^{n}, t^{n+1}\right), \\
\mathbf{C}\left(t^{n}\right)=\mathbf{C}^{n+1 / 3} ; \mathbf{C}^{n+2 / 3}=\mathbf{C}\left(t^{n+1}\right),
\end{array}\right. \\
& \left\{\begin{array}{l}
\frac{\partial \mathbf{C}}{\partial t}=\frac{f\left(\mathbf{C}^{n+2 / 3}\right)}{\lambda_{1}} \mathbf{I} \text { in } \Omega \times\left(t^{n}, t^{n+1}\right), \\
\mathbf{C}\left(t^{n}\right)=\mathbf{C}^{n+2 / 3} ; \mathbf{C}^{n+1}=\mathbf{C}\left(t^{n+1}\right) .
\end{array}\right.
\end{aligned}
$$

We have derived the following two equivalent equations based on the factorization approach with $s=$ $f\left(\mathbf{C}^{n+1 / 3}\right)$ for equations (26) and (27):

Lemma 2.1. For a matrix $\mathbf{A}$ and $\mathbf{C}=\mathbf{A} \mathbf{A}^{t}$, given the velocity $\mathbf{u}, \lambda_{1}(>0)$ and a real-valued constant $s$, (a). If $\mathbf{A}$ satisfies the equation $\frac{\partial \mathbf{A}}{\partial t}+(\mathbf{u} \cdot \boldsymbol{\nabla}) \mathbf{A}=\mathbf{0}$, then $\mathbf{C}$ satisfies the equation

$$
\frac{\partial \mathbf{C}}{\partial t}+(\mathbf{u} \cdot \nabla) \mathbf{C}=\mathbf{0}
$$

(b). If $\mathbf{A}$ satisfies the equation $\frac{\partial \mathbf{A}}{\partial t}+\frac{s}{2 \lambda_{1}} \mathbf{A}-(\boldsymbol{\nabla u}) \mathbf{A}=\mathbf{0}$, then $\mathbf{C}$ satisfies the equation

$$
\frac{\partial \mathbf{C}}{\partial t}+\frac{s}{\lambda_{1}} \mathbf{C}-(\boldsymbol{\nabla u}) \mathbf{C}-\mathbf{C}(\boldsymbol{\nabla} \mathbf{u})^{t}=\mathbf{0}
$$

Proof: (a) Multiplying by $\mathbf{A}^{t}$ on the right the first equation verified by $\mathbf{A}$, and then, similarly, multiplying by $\mathbf{A}$ on the left the transpose of the above equation, we obtain

$$
\begin{aligned}
& \frac{\partial \mathbf{A}}{\partial t} \mathbf{A}^{t}+(\mathbf{u} \cdot \boldsymbol{\nabla}) \mathbf{A} \mathbf{A}^{t}=\mathbf{0}, \\
& \mathbf{A} \frac{\partial \mathbf{A}^{t}}{\partial t}+\mathbf{A}(\mathbf{u} \cdot \boldsymbol{\nabla}) \mathbf{A}^{t}=\mathbf{0},
\end{aligned}
$$


Adding (L1) and (L2) gives,

$$
\frac{\partial \mathbf{A} \mathbf{A}^{t}}{\partial t}+(\mathbf{u} \cdot \boldsymbol{\nabla})\left(\mathbf{A} \mathbf{A}^{t}\right)=\mathbf{0} ; \text { that is, } \frac{\partial \mathbf{C}}{\partial t}+(\mathbf{u} \cdot \nabla)(\mathbf{C})=\mathbf{0} .
$$

(b) Doing as in (a) with the second equation verified by $\mathbf{A}$, we obtain

$$
\begin{gathered}
\frac{\partial \mathbf{A}}{\partial t} \mathbf{A}^{t}+\frac{s}{2 \lambda_{1}} \mathbf{A} \mathbf{A}^{t}-(\nabla \mathbf{u}) \mathbf{A} \mathbf{A}^{t}=\mathbf{0} \\
\mathbf{A} \frac{\partial \mathbf{A}^{t}}{\partial t}+\frac{s}{2 \lambda_{1}} \mathbf{A} \mathbf{A}^{t}-\mathbf{A} \mathbf{A}^{t}(\nabla \mathbf{u})^{t}=\mathbf{0}
\end{gathered}
$$

Adding (L3) and (L4) gives,

$$
\frac{\partial \mathbf{A} \mathbf{A}^{t}}{\partial t}+\frac{s}{\lambda_{1}} \mathbf{A} \mathbf{A}^{t}-(\nabla \mathbf{u}) \mathbf{A} \mathbf{A}^{t}-\mathbf{A} \mathbf{A}^{t}(\nabla \mathbf{u})^{t}=\mathbf{0}
$$

or,

$$
\frac{\partial \mathbf{C}}{\partial t}+\frac{s}{\lambda_{1}} \mathbf{C}-(\boldsymbol{\nabla u}) \mathbf{C}-\mathbf{C}(\boldsymbol{\nabla} \mathbf{u})^{t}=\mathbf{0} .
$$

Similarly, we can define finite dimensional spaces $\mathbf{V}_{\mathbf{A}_{L h}(t)}$ and $\mathbf{V}_{\mathbf{A}_{0 h}}$ for $\mathbf{A}$. When applying the Lie scheme to the discrete analogue of the problem (14)-(21), using the above Lozinski-Owens factorization of the conformation tensor $\mathbf{C}$, we obtain

$$
\mathbf{u}^{0}=\mathbf{u}_{0 h}, \mathbf{C}^{0}=\mathbf{C}_{0 h}, \mathbf{G}^{0}=\mathbf{G}_{0}, \mathbf{V}^{0}=\mathbf{V}_{0}, \omega^{0}=\omega_{0} \text { given. }
$$

For $n \geq 0, \mathbf{u}^{n}, \mathbf{C}^{n}, \mathbf{G}^{n}, \mathbf{V}^{n}, \omega^{n}$ being known, we compute $\mathbf{u}^{n+\frac{1}{5}}$, and $p^{n+\frac{1}{5}}$ via the solution of

$$
\left\{\begin{array}{l}
\rho_{f} \int_{\Omega} \frac{\mathbf{u}^{n+\frac{1}{5}}-\mathbf{u}^{n}}{\triangle t} \cdot \mathbf{v} d \mathbf{x}-\int_{\Omega} p^{n+\frac{1}{5}} \nabla \cdot \mathbf{v} d \mathbf{x}=0, \forall \mathbf{v} \in \mathbf{V}_{0 h} \\
\int_{\Omega} q \boldsymbol{\nabla} \cdot \mathbf{u}^{n+\frac{1}{5}} d \mathbf{x}=0, \forall q \in L_{h}^{2} ; \mathbf{u}^{n+\frac{1}{5}} \in \mathbf{V}_{\mathbf{g}_{0 h}^{n+1}}^{n}, p^{n+\frac{1}{5}} \in L_{0 h}^{2}
\end{array}\right.
$$

Next, we compute $\mathbf{u}^{n+\frac{2}{5}}$ and $\mathbf{A}^{n+\frac{2}{5}}$ via the solution of

$$
\begin{aligned}
& \left\{\begin{array}{l}
\rho_{f} \int_{\Omega} \frac{\partial \mathbf{u}(t)}{\partial t} \cdot \mathbf{v} d \mathbf{x}+\int_{\Omega}\left(\mathbf{u}^{n+\frac{1}{5}} \cdot \nabla\right) \mathbf{u}(t) \cdot \mathbf{v} d \mathbf{x}=0, \forall \mathbf{v} \in \mathbf{V}_{0 h}^{n+1,-}, \\
\mathbf{u}\left(t^{n}\right)=\mathbf{u}^{n+\frac{1}{5}} \\
\mathbf{u}(t) \in \mathbf{V}_{h}, \mathbf{u}(t)=\mathbf{g}_{0 h}\left(t^{n+1}\right) \text { on } \Gamma^{n+1,-} \times\left[t^{n}, t^{n+1}\right]
\end{array}\right. \\
& \left\{\begin{array}{l}
\int_{\Omega} \frac{\partial \mathbf{A}(t)}{\partial t}: \mathbf{s} d \mathbf{x}+\int_{\Omega}\left(\mathbf{u}^{n+\frac{1}{5}} \cdot \nabla\right) \mathbf{A}(t): \mathbf{s} d \mathbf{x}=0, \forall \mathbf{s} \in \mathbf{V}_{\mathbf{A}_{0 h}+1}, \\
\mathbf{A}\left(t^{n}\right)=\mathbf{A}^{n}, \text { where } \mathbf{A}^{n}\left(\mathbf{A}^{n}\right)^{t}=\mathbf{C}^{n} \\
\mathbf{A}(t) \in \mathbf{V}_{\mathbf{A}_{L h}}^{n+1}, t \in\left[t^{n}, t^{n+1}\right]
\end{array}\right.
\end{aligned}
$$

and set $\mathbf{u}^{n+\frac{2}{5}}=\mathbf{u}\left(t^{n+1}\right)$ and $\mathbf{A}^{n+\frac{2}{5}}=\mathbf{A}\left(t^{n+1}\right) ;$ above $\Gamma^{n+1,-}=\left\{\mathbf{x} \in \Gamma, \mathbf{g}_{0 h}\left(t^{n+1}\right)(\mathbf{x}) \cdot \mathbf{n}(\mathbf{x})<0\right\}, \mathbf{V}_{h}=$ $\left\{\mathbf{v}_{h}\left|\mathbf{v}_{h} \in\left(C^{0}(\bar{\Omega})\right)^{2}, \mathbf{v}_{h}\right|_{E} \in\left(P_{1}\right)^{2}, \forall E \in \mathcal{T}_{h},\right\}$, and $\mathbf{V}_{0 h}^{n+1,-}=\left\{\mathbf{v} \in \mathbf{V}_{h}, \mathbf{v}=0\right.$, on $\left.\Gamma^{n+1,-}\right\}$.

Then, compute $\mathbf{u}^{n+\frac{3}{5}}$ and $\mathbf{A}^{n+\frac{3}{5}}$ via the solution of

$$
\left\{\begin{array}{l}
\rho_{f} \int_{\Omega} \frac{\mathbf{u}^{n+\frac{3}{5}}-\mathbf{u}^{n+\frac{2}{5}}}{\triangle t} \cdot \mathbf{v} d \mathbf{x}+\alpha \mu \int_{\Omega} \nabla \mathbf{u}^{n+\frac{3}{5}}: \nabla \mathbf{v} d \mathbf{x}=0 \\
\forall \mathbf{v} \in \mathbf{V}_{0 h} ; \mathbf{u}^{n+\frac{3}{5}} \in \mathbf{V}_{\mathbf{g}_{0 h}}^{n+1}
\end{array}\right.
$$




$$
\left\{\begin{array}{l}
\int_{\Omega}\left(\frac{\mathbf{A}^{n+\frac{3}{5}}-\mathbf{A}^{n+\frac{2}{5}}}{\triangle t}-\left(\nabla \mathbf{u}^{n+\frac{3}{5}}\right) \mathbf{A}^{n+\frac{3}{5}}+\frac{f\left(\mathbf{A}^{n+\frac{2}{5}}\left(\mathbf{A}^{n+\frac{2}{5}}\right)^{t}\right)}{2 \lambda_{1}} \mathbf{A}^{n+\frac{3}{5}}\right): \mathbf{s} d \mathbf{x}=0 \\
\forall \mathbf{s} \in \mathbf{V}_{\mathbf{A}_{0 h}}^{n+1} ; \mathbf{A}^{n+\frac{3}{5}} \in \mathbf{V}_{\mathbf{A}_{L h}^{n+1}}^{n+1}
\end{array}\right.
$$

and set

$$
\mathbf{C}^{n+\frac{3}{5}}=\mathbf{A}^{n+\frac{3}{5}}\left(\mathbf{A}^{n+\frac{3}{5}}\right)^{t}+\frac{\triangle t f\left(\mathbf{A}^{n+\frac{3}{5}}\left(\mathbf{A}^{n+\frac{3}{5}}\right)^{t}\right)}{\lambda_{1}} \mathbf{I} .
$$

Then, predict the position and the translation velocity of the center of mass as follows.

Take $\mathbf{V}^{n+\frac{3}{5}, 0}=\mathbf{V}^{n}$ and $\mathbf{G}^{n+\frac{3}{5}, 0}=\mathbf{G}^{n}$; then predict the new position and translation velocity via the following sub-cycling and predicting-correcting technique

For $k=1,2, \ldots, N$, compute

$$
\begin{aligned}
\hat{\mathbf{V}}^{n+\frac{3}{5}, k} & =\mathbf{V}^{n+\frac{3}{5}, k-1}+\left(1-\rho_{f} / \rho_{s}\right)^{-1} M_{p}^{-1} \mathbf{F}^{r}\left(\mathbf{G}^{n+\frac{3}{5}, k-1}\right) \triangle t / 2 N, \\
\hat{\mathbf{G}}^{n+\frac{3}{5}, k} & =\mathbf{G}^{n+\frac{3}{5}, k-1}+(\triangle t / 4 N)\left(\hat{\mathbf{V}}^{n+\frac{3}{5}, k}+\mathbf{V}^{n+\frac{3}{5}, k-1}\right), \\
\mathbf{V}^{n+\frac{3}{5}, k} & =\mathbf{V}^{n+\frac{3}{5}, k-1}+\left(1-\rho_{f} / \rho_{s}\right)^{-1} M_{p}^{-1}\left(\mathbf{F}^{r}\left(\hat{\mathbf{G}}^{n+\frac{3}{5}, k}\right)+\mathbf{F}^{r}\left(\mathbf{G}^{n+\frac{3}{5}, k-1}\right)\right) \triangle t / 4 N, \\
\mathbf{G}^{n+\frac{3}{5}, k} & =\mathbf{G}^{n+\frac{3}{5}, k-1}+(\triangle t / 4 N)\left(\mathbf{V}^{n+\frac{3}{5}, k}+\mathbf{V}^{n+\frac{3}{5}, k-1}\right),
\end{aligned}
$$

end do; let $\mathbf{V}^{n+\frac{3}{5}}=\mathbf{V}^{n+\frac{3}{5}, N}, \mathbf{G}^{n+\frac{3}{5}}=\mathbf{G}^{n+\frac{3}{5}, N}$.

Next compute $\left\{\mathbf{u}^{n+\frac{4}{5}}, \boldsymbol{\lambda}^{n+\frac{4}{5}}, \mathbf{V}^{n+\frac{4}{5}}, \omega^{n+\frac{4}{5}}\right\}$ via the solution of

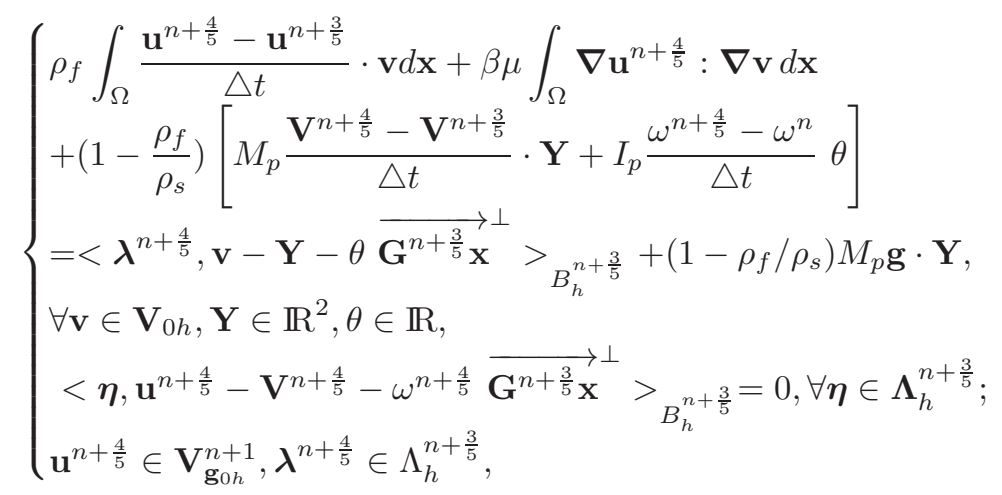

and set $\mathbf{C}^{n+\frac{4}{5}}=\mathbf{C}^{n+\frac{3}{5}}$, and then let $\mathbf{C}^{n+\frac{4}{5}}=\mathbf{I}$ in $B_{h}^{n+\frac{3}{5}}$.

Then take $\mathbf{V}^{n+1,0}=\mathbf{V}^{n+\frac{4}{5}}$ and $\mathbf{G}^{n+1,0}=\mathbf{G}^{n+\frac{3}{5}}$; and predict the final position and translation velocity as follows:

For $k=1,2, \ldots, N$, compute

$$
\begin{aligned}
& \hat{\mathbf{V}}^{n+1, k}=\mathbf{V}^{n+1, k-1}+\left(1-\rho_{f} / \rho_{s}\right)^{-1} M_{p}^{-1} \mathbf{F}^{r}\left(\mathbf{G}^{n+1, k-1}\right) \triangle t / 2 N, \\
& \hat{\mathbf{G}}^{n+1, k}=\mathbf{G}^{n+1, k-1}+(\triangle t / 4 N)\left(\hat{\mathbf{V}}^{n+1, k}+\mathbf{V}^{n+1, k-1}\right), \\
& \mathbf{V}^{n+1, k}=\mathbf{V}^{n+1, k-1}+\left(1-\rho_{f} / \rho_{s}\right)^{-1} M_{p}^{-1}\left(\mathbf{F}^{r}\left(\hat{\mathbf{G}}^{n+1, k}\right)+\mathbf{F}^{r}\left(\mathbf{G}^{n+1, k-1}\right)\right) \triangle t / 4 N, \\
& \mathbf{G}^{n+1, k}=\mathbf{G}^{n+1, k-1}+(\triangle t / 4 N)\left(\mathbf{V}^{n+1, k}+\mathbf{V}^{n+1, k-1}\right),
\end{aligned}
$$

end do; let $\mathbf{V}^{n+1}=\mathbf{V}^{n+1, N}, \mathbf{G}^{n+1}=\mathbf{G}^{n+1, N}$.

Finally, compute $\mathbf{u}^{n+1}$ via the solution of

$$
\left\{\begin{array}{l}
\rho_{f} \int_{\Omega} \frac{\mathbf{u}^{n+1}-\mathbf{u}^{n+\frac{4}{5}}}{\Delta t} \cdot \mathbf{v} d \mathbf{x}+\gamma \mu \int_{\Omega} \nabla \mathbf{u}^{n+1}: \nabla \mathbf{v} d \mathbf{x} \\
=\frac{\eta}{\lambda_{1}} \int_{\Omega} \mathbf{v} \cdot\left(\boldsymbol{\nabla} \cdot f\left(\mathbf{C}^{n+\frac{4}{5}}\right)\left(\mathbf{C}^{n+\frac{4}{5}}-\mathbf{I}\right)\right) d \mathbf{x}, \forall \mathbf{v} \in \mathbf{V}_{0 h} ; \mathbf{u}^{n+1} \in \mathbf{V}_{\mathbf{g}_{0 h}^{n+1}}
\end{array}\right.
$$


We complete the final step by setting $\mathbf{C}^{n+1}=\mathbf{C}^{n+\frac{4}{5}}$, and $\omega^{n+1}=\omega^{n+\frac{4}{5}}$.

In the above, $\mathbf{u}_{0 h}$ is an approximation of $\mathbf{u}_{0}$ so that $\int_{\Omega} q \boldsymbol{\nabla} \cdot \mathbf{u}_{0 h} d \mathbf{x}=0, \forall q \in L_{h}^{2}, \mathbf{V}_{\mathbf{g}_{0 h}}^{n+1}=\mathbf{V}_{\mathbf{g}_{0 h}\left(t^{n+1}\right)}$, $\Lambda_{h}^{n+s}=\Lambda_{h}\left(t^{n+s}\right), \mathbf{V}_{\mathbf{A}_{L h}}^{n+1}=\mathbf{V}_{\mathbf{A}_{L h}\left(t^{n+1}\right)}, B_{h}^{n+s}=B_{h}\left(t^{n+s}\right)$, the spaces $\mathbf{V}_{\mathbf{A}_{L_{h}}(t)}$ and $\mathbf{V}_{\mathbf{A}_{0 h}(t)}$ for $\mathbf{A}$ being defined similar to those for $\mathbf{V}_{\mathbf{C}_{L_{h}}(t)}$ and $\mathbf{V}_{\mathbf{C}_{0 h}(t)}$, and $\alpha+\beta+\gamma=1$, for $\alpha, \beta, \gamma \geq 0$.

\subsection{Solution strategies}

Applying scheme (29)-(45) to the solution of system (14)-(21) leads to a sequence of simpler sub-problems, namely: (i) the (degenerated) discrete Stokes problem (30) where one forces the incompressibility condition, (ii) the two advection sub-problems (31) and (32), associated with the velocity and the conformation tensor, respectively, (iii) the velocity related diffusion sub-problem (33), and the updating of the conformation tensor via (34) and (35), (iv) system (36)-(39) to predict the new position of the articles, (v) system (40) to force rigid body motion inside the particle and update the translation and angular velocity of the particle (forcing $\mathbf{C}=\mathbf{I}$ inside the particle is also done via this 5th fractional step), (vi) system (41)-(44) to improve the positioning of the particle, and finally (vii) the velocity related diffusion sub-problem (45) taking into account the effect of the updated value of the polymeric stress tensor.

The resulting methodology is quite modular and easy to implement. The degenerated quasi-Stokes problem (30) is solved by an Uzawa/ preconditioned conjugate gradient algorithm operating in the space $L_{0 h}^{2}$ as discussed in [11] and [19]. The advection problems (31) and (32) are solved by a wave-like equation method (see, e.g., [19], [22], and [11] (p. 102)) which is a numerical dissipation free explicit method. Since the advection problem is decoupled from the other ones, we can choose a proper sub-time step so that the CFL condition is satisfied. Problem (40), concerning the rigid body motion enforcement, is a saddle point problem and is solved by a conjugate gradient method discussed in, e.g., [11] and [19]. Concerning the constraint, $\mathbf{C}=\mathbf{I}$ in $B$, we have chosen a simple approach to deal with such constraint in scheme (29)-(45) by setting $\mathbf{C}^{n+\frac{4}{5}}=\mathbf{I}$ in $B_{h}^{n+\frac{3}{5}}$. Problems (33) and (45) are classical elliptic problems which can be solved by a matrix-free fast solver. Concerning (36)-(39) and (41)-(44), we use a predictor-corrector scheme to obtain the position of the mass center and the translation velocity of the particle. Problem (34) reduces to a simple algebraic equation at each grid point which can be solved easily if we use the trapezoidal quadrature rule to compute the integrals as in [11].

Remark 2.1. Other schemes relying on the DLM/FD formulation have been used for the simulation of Oldroyd-B particulate flow, in [16] and [17] in particular: In [16], a sophisticated third-order upwind-biased positive only scheme [23] was used to preserve the positivity of the conformation tensor. In [17], the DLM/FD method was modified, so that it could handle $Q_{1}-P_{0}$ rectangular finite element approximations of the velocity and the pressure, a third-order upwind-biased finite difference scheme being used to discretize the constitutive equation verified by the conformation tensor.

\section{Numerical results and discussion}

To study the effect of the polymer extension limit coefficient $L$ on the particle chain formation while settling, we have considered the cases of two, three and six disks settling in a viscoelastic fluid as in [11] since vertical chains are known to be formed for these cases. The computational results for disks settling in Oldroyd-B fluid are obtained by the numerical method developed in [11] and these results are compared with those obtained by the scheme discussed in the previous section for the FENE-CR model at large values of $L$ for validation purpose since $f(\mathbf{C})=\frac{L^{2}}{L^{2}-\operatorname{tr}(\mathbf{C})} \rightarrow 1$ as $L \rightarrow \infty$ (i.e., the FENE-CR model has almost recovered the Oldroyd-B model for large values of $L$ ). In the following discussion, the particle Reynolds number is $\operatorname{Re}=\frac{\rho_{f} U d}{\eta_{1}}$, the Deborah number is $\mathrm{De}=\frac{\lambda_{1} U}{d}$, the Mach number is $\mathrm{M}=\sqrt{\mathrm{DeRe}}$, and the elasticity 

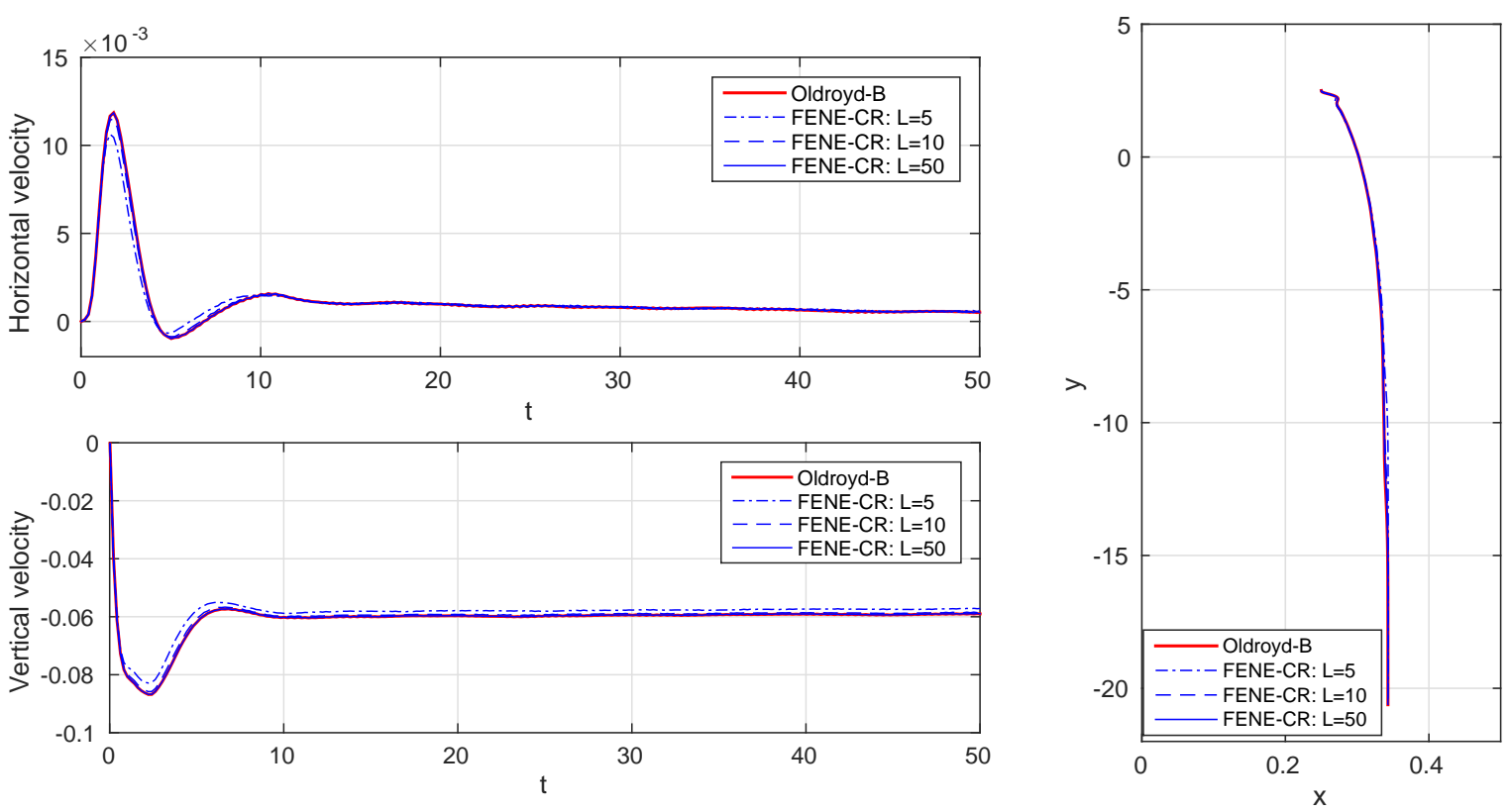

Figure 2: Histories of the particle horizontal velocity (left top), vertical velocity (left bottom) and trajectories of the disk (right) for $\lambda_{1}=2.025$ (the associated numbers are $\mathrm{Re}=0.4186, \mathrm{M}=0.4393$, $\mathrm{De}=0.4611, \mathrm{E}=1.1016$ for the Oldroyd-B fluid and $\mathrm{Re}=0.4059, \mathrm{M}=0.4261, \mathrm{De}=0.4472, \mathrm{E}=1.1016$ for the FENE-CR fluid with $L=5)$.
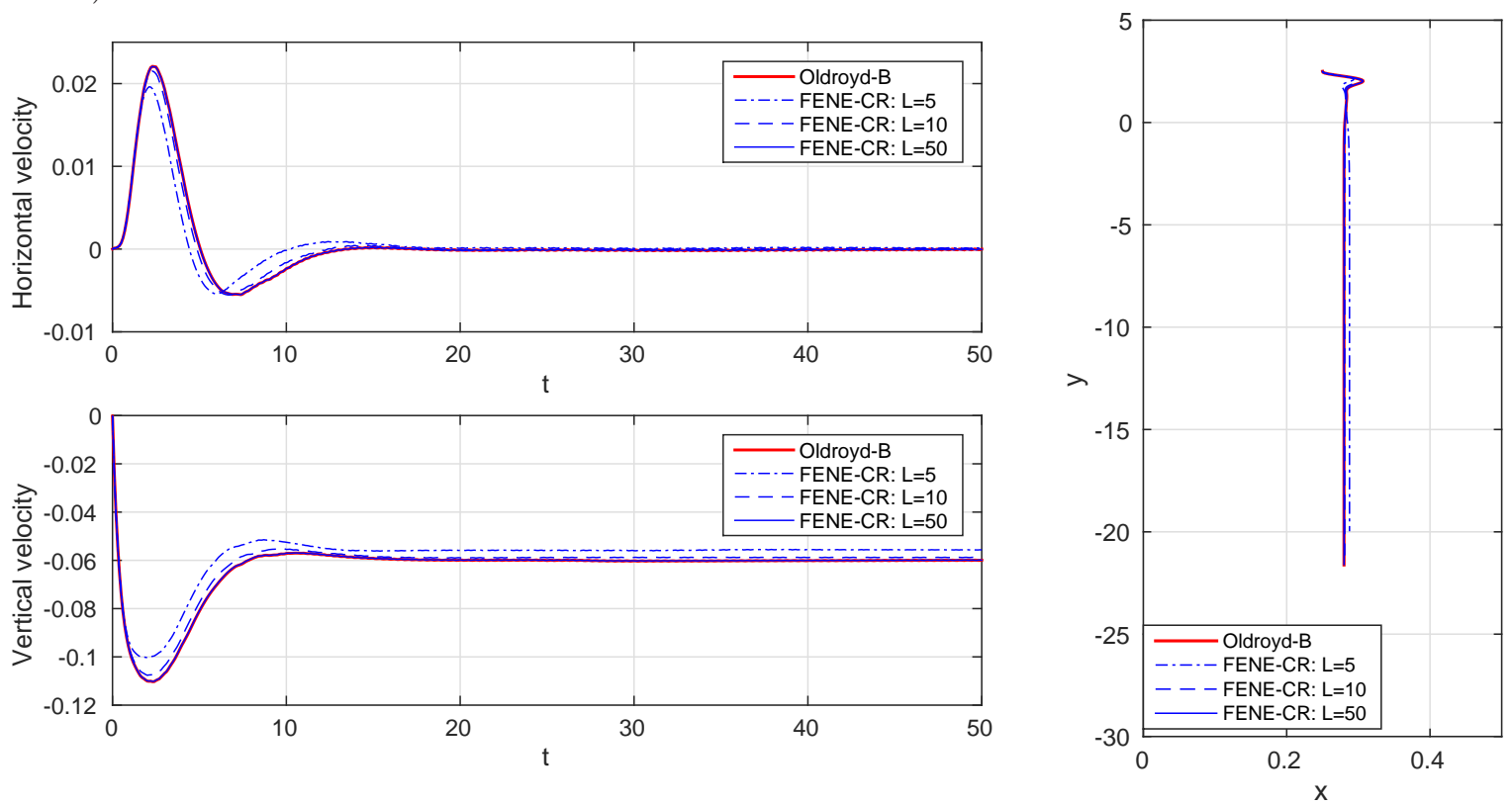

Figure 3: Histories of the particle horizontal velocity (left top), vertical velocity (left bottom) and trajectories of the disk (right) for $\lambda_{1}=4.05$ (the associated numbers are $\mathrm{Re}=0.4403, \mathrm{M}=0.6536$, $\mathrm{De}=0.9701, \mathrm{E}=2.2032$ for the Oldroyd-B fluid and $\mathrm{Re}=0.4110, \mathrm{M}=0.6101, \mathrm{De}=0.9056, \mathrm{E}=2.2032$ for the FENE-CR fluid with $\mathrm{L}=5$ ). 

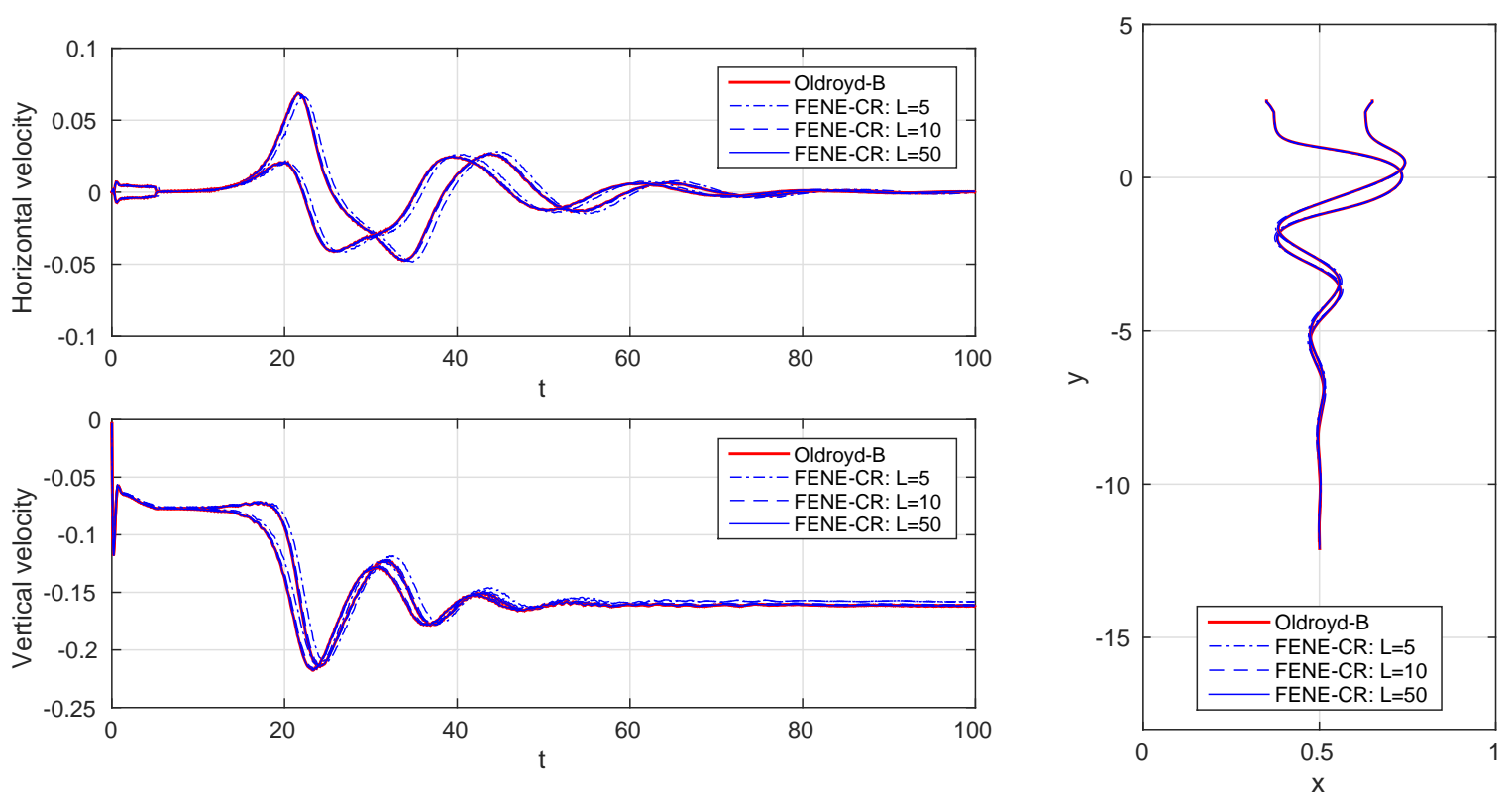

Figure 4: Histories of the particle horizontal velocity (left top), vertical velocity (left bottom) and trajectories of the two disks (right) for $\lambda_{1}=0.5$ (the associated numbers are $\mathrm{Re}=0.202, \mathrm{M}=0.255, \mathrm{De}=0.323, \mathrm{E}=1.6$ and $\mathrm{Re}=0.197, \mathrm{M}=0.249, \mathrm{De}=0.316, \mathrm{E}=1.6$ for the Oldroyd-B fluid and the FENE-CR with $L=5$, respectively).

number is $\mathrm{E}=\mathrm{De} / \mathrm{Re}=\frac{\lambda_{1} \eta_{1}}{d^{2} \rho_{f}}$; above $U$ is the averaged terminal speed of the disks and $d$ is the disk diameter. We assume that all dimensional quantities are in the CGS units in this section.

\subsection{Settling of 1,2 and 3 disks}

We have considered first the settling of one disk in a vertical channel of infinite length filled with a viscoelastic fluid as in [11]. The computational domain is $\Omega=(0,1) \times(0,6)$ initially, then it moves vertically with the mass center of the disk (see, e.g., [24] and [25] and references therein for adjusting the computational domain according to the position of the particle). The disk diameter is $d=0.25$ and the initial position of the disk center is at $(0.25,2.5)$. The disk density $\rho_{s}$ is 1.0007 and the fluid density $\rho_{f}$ is 1 . The fluid viscosity $\eta_{1}$ is 0.034 . The relaxation time $\lambda_{1}$ is either 2.025 or 4.05 and the retardation time $\lambda_{2}$ is $\lambda_{1} / 8$. Hence the values of the elasticity number E are 1.1016 and 2.2032 for $\lambda_{1}=2.025$ and 4.05 , respectively. The maximal polymer extension $L$ is either 5, 10, or 50 for the FENE-CR model. The mesh sizes for the velocity field, conformation tensor and pressure are $h=1 / 128,1 / 128$, and 1/64, respectively, the time step being 0.0004 . Fig. 2 shows that the trajectories of a disk settling in either Oldroyd-B or FENE-CR fluids are almost identical for $L=5$, 10 , and 50 and $\lambda_{1}=1.1016(\mathrm{E}=1.1016)$; however in Fig. 3 the disk trajectory for $L=5$ and $\lambda_{1}=4.05$ is quite different from those for $L=10$ and 50 and $\lambda_{1}=4.05$ and from the Oldroyd-B associated one. For $\lambda_{1}=4.05$ $(\mathrm{E}=2.2032)$, the disk trajectory for $L=5$ is different from the others is a consequence of the fact that its Deborah number is slightly smaller (actually the values of the associated De are 0.9056, 0.9519, 0.9693, and 0.9701 for the FENE-CR fluid for $L=5,10,50$, and also for the Oldroyd-B one). As observed in [26], the effect of the Deborah number on the single particle settling trajectory is that the smaller De is, the closer to the center line the equilibrium position is. The histories of the disk horizontal and vertical velocities, and the trajectory of its center, show that the larger is the extension coefficient $L$, the closer are these velocities and trajectory to those associated with the corresponding Oldroyd-B fluid. 

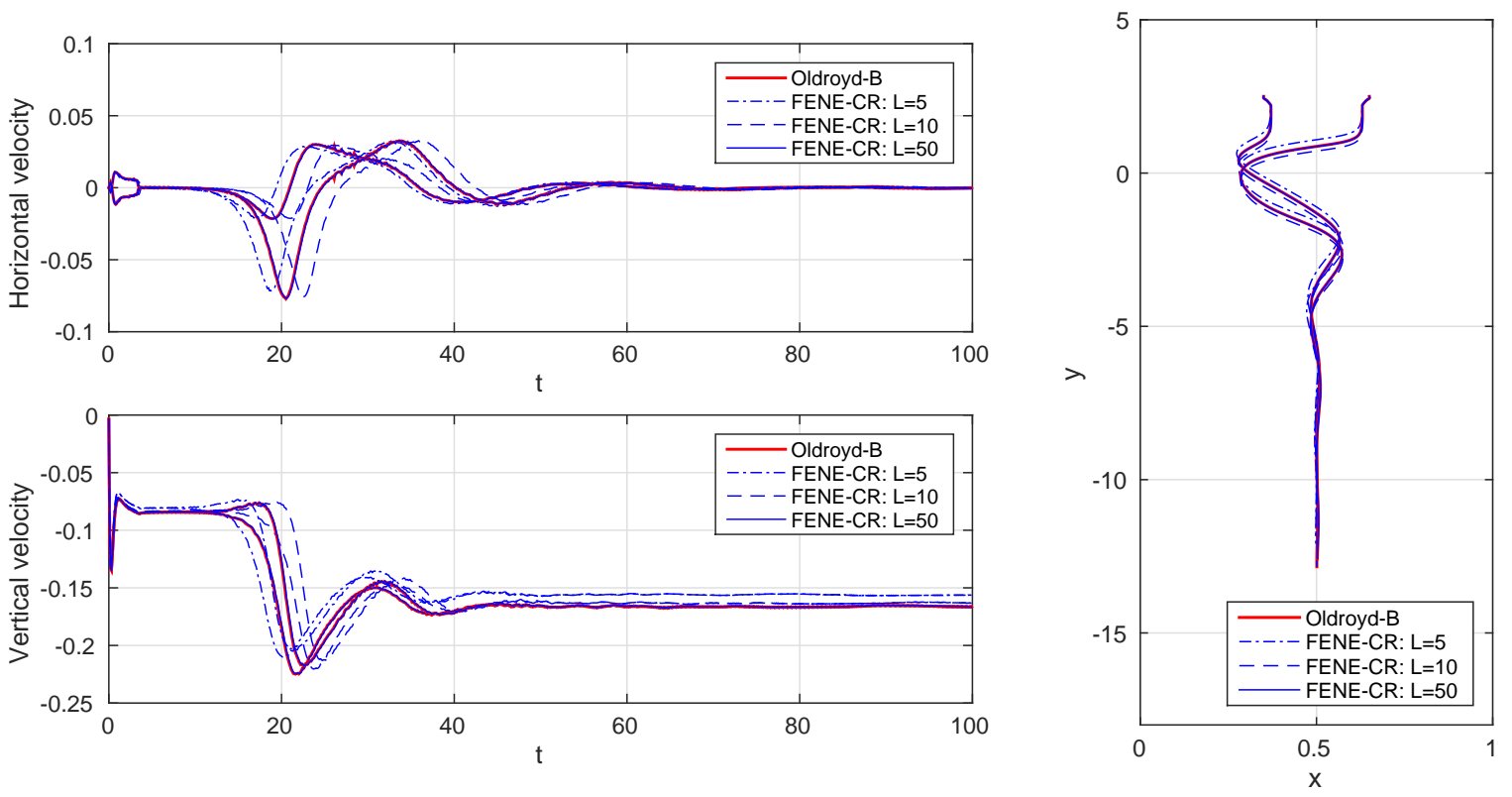

Figure 5: Histories of the particle horizontal velocity (left top), vertical velocity (left bottom) and trajectories of the two disks (right) for $\lambda_{1}=1$ (the associated numbers are $\mathrm{Re}=0.208, \mathrm{M}=0.371, \mathrm{De}=0.664, \mathrm{E}=3.2$ and $\mathrm{Re}=0.195, \mathrm{M}=0.35, \mathrm{De}=0.625, \mathrm{E}=3.2$ for the Oldroyd-B fluid and the FENE-CR with $L=5$, respectively).
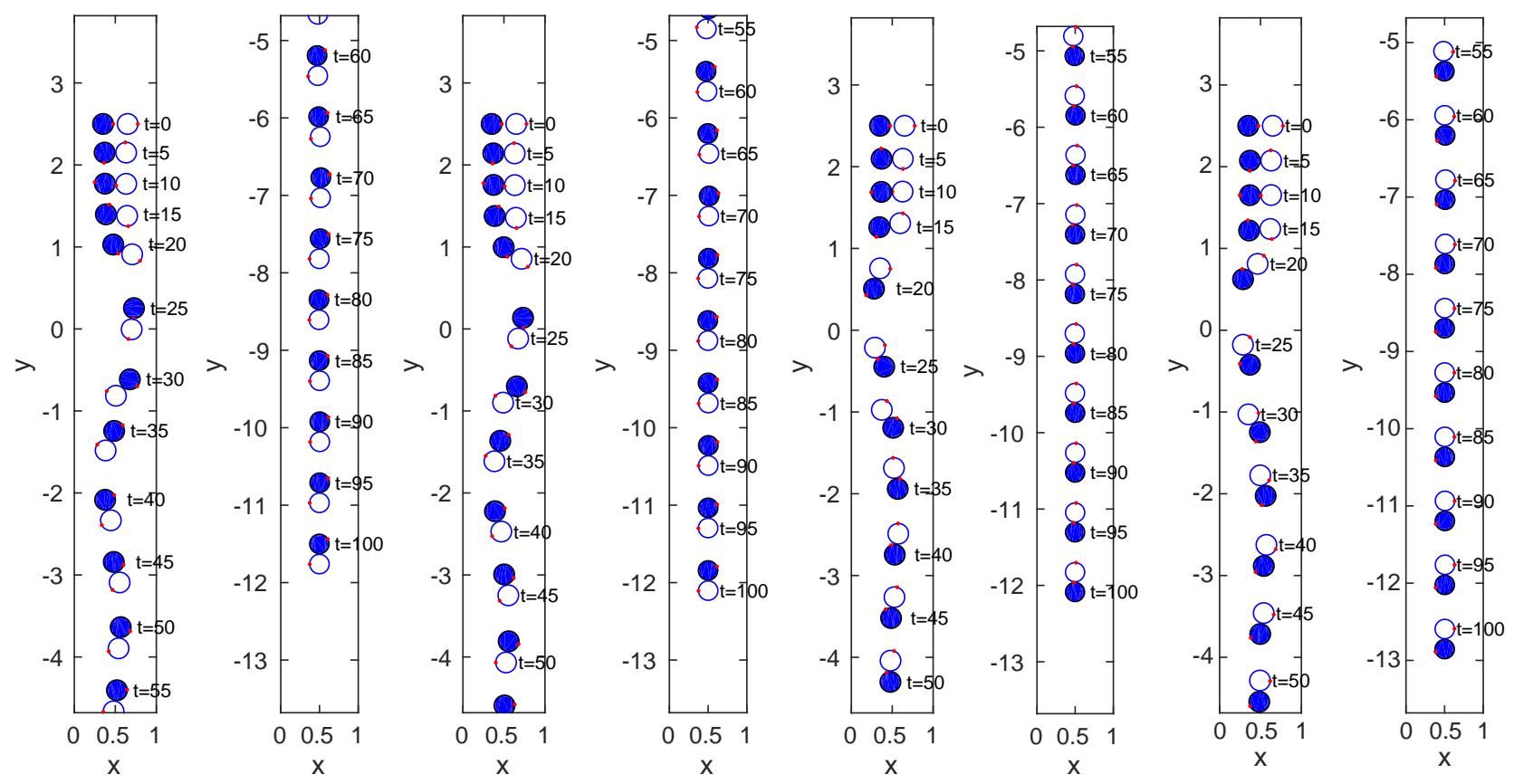

Figure 6: Positions of the two disks : FENE-CR with $L=5$ and $\lambda_{1}=0.5$ (left two), Oldroyd-B and $\lambda_{1}=0.5$ (middle left two), FENE-CR with $L=5$ and $\lambda_{1}=1$ (middle right two), and Oldroyd-B and $\lambda_{1}=1$ (right two). 

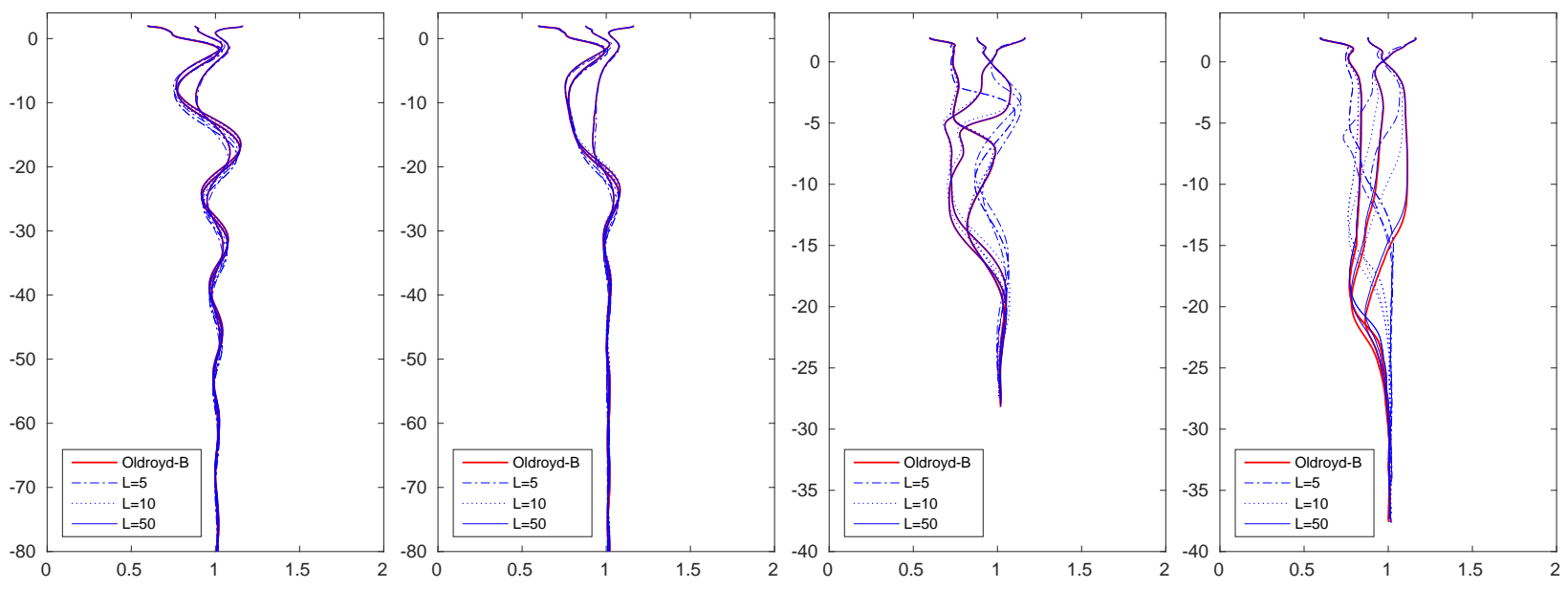

Figure 7: The disk trajectories: $\rho_{s}=1.0075$ and $\lambda_{1}=0.75$ (left), $\rho_{s}=1.01, \lambda_{1}=0.75$ (middle left), $\rho_{s}=1.0075$, $\lambda_{1}=1.5$ (middle right), and $\rho_{s}=1.01 \lambda_{1}=1.5$ (right).

For those cases where two disks are sedimenting in a vertical channel of infinite length filled with a viscoelastic fluid, the computational domain is $\Omega=(0,1) \times(0,6)$ initially and then it moves vertically with the mass center of the lowest of the two disks. The two disk diameters are $d=0.25$ and the initial position of the disk centers are at $(0.35,2.5)$ and $(0.65,2.5)$, respectively. The disk density $\rho_{s}$ is 1.01 and the fluid density $\rho_{f}$ is 1 . The fluid viscosity $\eta_{1}$ is 0.2 . The relaxation time $\lambda_{1}$ is either 0.5 or 1 and the retardation time $\lambda_{2}$ is $\lambda_{1} / 4$. Hence the elasticity numbers $\mathrm{E}$ are 1.6 and 3.2 for $\lambda_{1}=0.5$ and 1 , respectively. The maximal polymer extension $L$ is either 5,10 , or 50 for the FENE-CR model. The mesh sizes for the velocity field, conformation tensor and pressure are $h=1 / 96,1 / 96$, and $1 / 48$, respectively, the time step being 0.0004. Fig. 4 shows that the trajectories of two disks settling in either Oldroyd-B or FENE-CR fluids are almost identical for $\lambda_{1}=0.5$ and $L=5,10$, and 50. Similar behaviors are also observed for the vertical and horizontal particle translation velocities shown in Figs. 4. But, in Fig. 5, the two disk trajectories for $\lambda_{1}=1$ and $L=5$ and 10 are slightly different from those for $\lambda_{1}=1$ and $L=50$ and those for the Oldroyd-B fluid. Similar behaviors for the cases of $L=5$ and 10 are also observed for the vertical and horizontal particle translation velocities shown in Fig. 5. For all the two disk cases considered here, the following scenario takes place: the two disks attract each other, forming a horizontal chain, then the chain rotates so that its broadside is aligned with the falling direction (the vertical one here); this behavior differs from the well known phenomenon called drafting, kissing and tumbling for disks settling in Newtonian fluid [27]. In the position snapshots shown in Fig. 6, for the case with $L=5$ and $\mathrm{E}=1.6$, the two disks move slower due to the smaller relaxation time and the smaller value of $L$. On the other hand, for the case of the Oldroyd-B fluid with $\mathrm{E}=3.2$, the two disks move further down in the channel. However, for the two cases with $L=50$, the particle motions are almost identical to those in the Oldroyd-B fluid as in Figs. 4 and 5.

For the cases of three disks sedimenting in a vertical channel of infinite length filled with a viscoelastic fluid, the computational domain is $\Omega=(0,2) \times(0,5)$ initially and then it moves vertically with the mass center of the lowest of the three disks. The three disk diameters are $d=0.25$ and the initial disk centers are $(0.6,2),(0.88,2)$ and $(1.16,2)$, respectively. The disk density $\rho_{s}$ is either 1.0075 or 1.01 and the fluid density $\rho_{f}$ is 1 . The fluid viscosity $\eta_{1}$ is 0.26 . The relaxation time $\lambda_{1}$ is either 0.75 or 1.5 and the retardation time $\lambda_{2}$ is $\lambda_{1} / 8$. Hence the elasticity number $\mathrm{E}$ are 3.12 and 6.24 for $\lambda_{1}=0.75$ and 1.5 , respectively. The maximal polymer extension $L$ is either 5,10 , or 50 for the FENE-CR model. The mesh sizes for the velocity field, conformation tensor and pressure are $h=1 / 96,1 / 96$, and $1 / 48$, respectively, the time step being 0.0004 . Fig. 

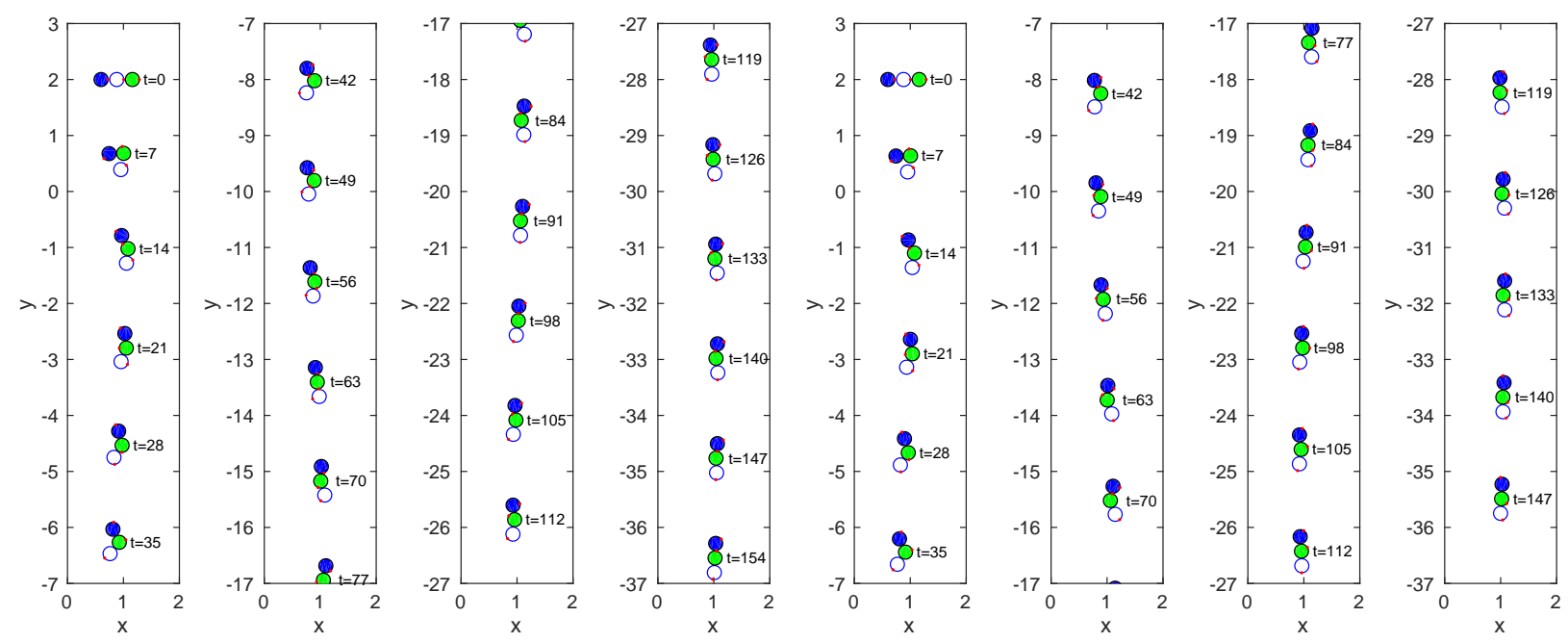

Figure 8: Positions of the three disks for $\rho_{s}=1.0075$ : FENE-CR with $L=5$ and $\lambda_{1}=0.75, \operatorname{Re}=0.244$, $\mathrm{M}=0.431, \mathrm{De}=0.762$, and $\mathrm{E}=3.12$ (left four) and Oldroyd-B with $\lambda_{1}=0.75, \mathrm{Re}=0.259, \mathrm{M}=0.440, \mathrm{De}=0.777$, $\mathrm{E}=3.12$ (right four).
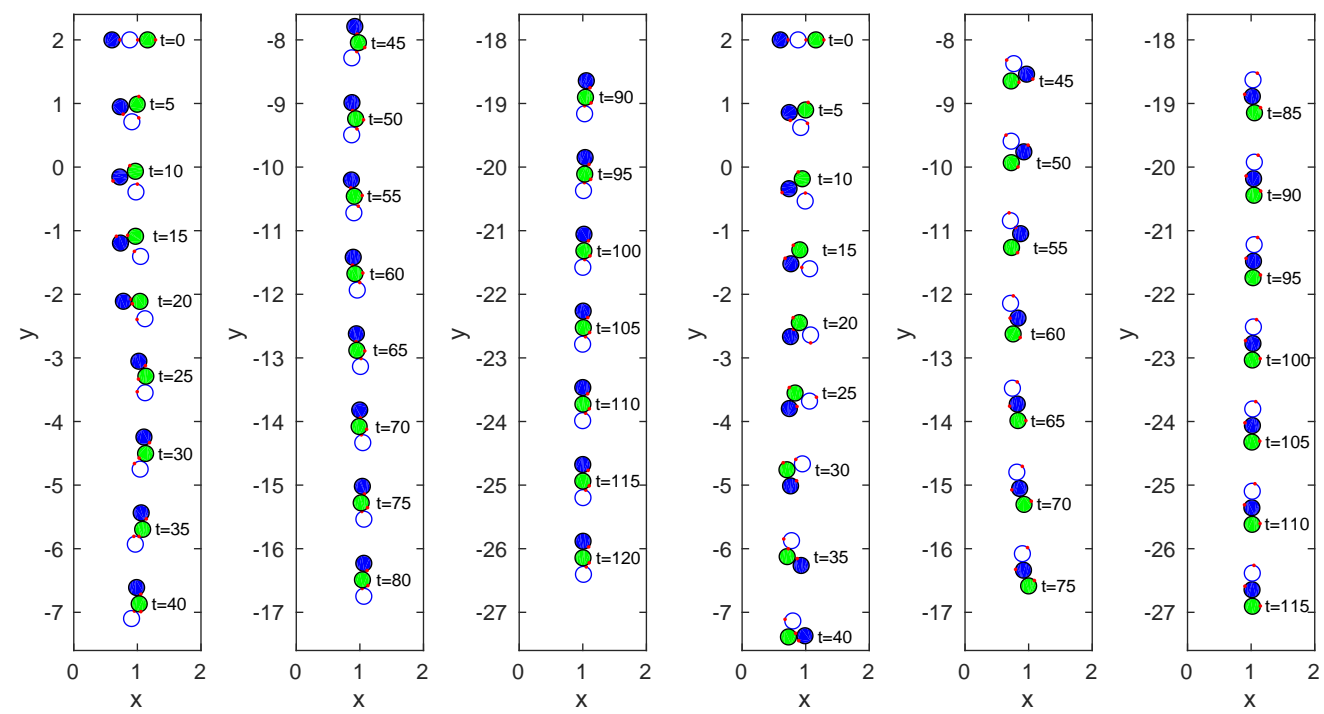

Figure 9: Positions of the three disks for $\rho_{s}=1.0075$ : FENE-CR with $L=5$ and $\lambda_{1}=1.5, \operatorname{Re}=0.232$, $\mathrm{M}=0.579, \mathrm{De}=1.447, \mathrm{E}=6.24$ (left three) and Oldroyd-B with $\lambda_{1}=1.5, \mathrm{Re}=0.248, \mathrm{M}=0.620, \mathrm{De}=1.550$, $\mathrm{E}=6.24$ (right three). 

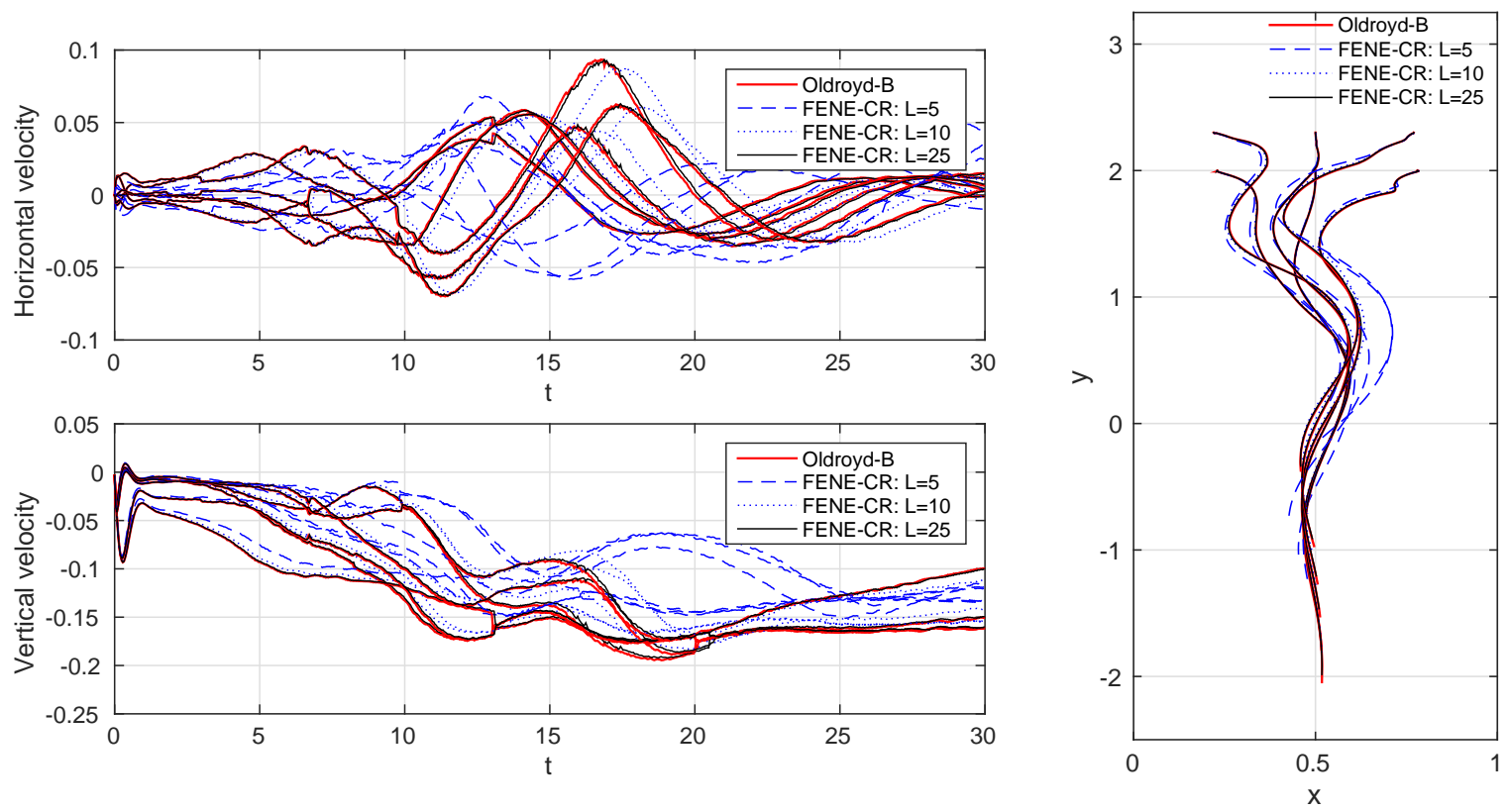

Figure 10: Histories of the particle velocity (left) and trajectories for six disks (right) for $\lambda_{1}=1.3$.

7 shows that the trajectories of the three disks of both densities settling in either Oldroyd-B or FENE-CR fluids are close to each other for $\mathrm{E}=3.12, L=10$ and 50 . But at $\mathrm{E}=6.24$, the three disk trajectories for $L=5$ are very different from those for $L=50$ and those for Oldroyd-B fluid. For all cases shown in Figs. 8 and 9 , the middle disk moves downward faster first and the other two are drafted toward this leading disk. Then they rearrange themselves and form a curved chain. The rearrangement of particles does depend on the fluid properties (e.g., the relaxation time) as shown in Figs. 8 and 9. Of course, the triangular mesh also has its effect due to its non-symmetric property. Finally the curved chain straighten out due to the large normal stress next to the middle disk in the curved chain (as discussed in [28]). For the cases where $L=50$, the particle trajectories are almost identical to those of the related Oldroyd-B fluid as in Fig. 7.

The above numerical results suggest that the effect of the shorter extensibility in the FENE-CR model on the particle settling trajectory can be enhanced by increasing the value of the relaxation time $\lambda_{1}$. It is known that when the elasticity number $\mathrm{E}=\mathrm{De} / \mathrm{Re}$ is larger than the critical value $(O(1))$ and the Mach number $\mathrm{M}=\sqrt{\mathrm{DeRe}}$ is less than the critical value, a long particle settling in an Oldroyd-B fluid can turn its broadside parallel to the falling direction ([28], [29]). For the two disk and three disk cases considered in this section, where the values of the elasticity number are larger than the critical values while those of the Mach number are smaller than 1, the particle chains are always formed with their "broadside" parallel to the flow direction. Thus, at least for the cases and the values of $L$ considered in this section, we observed that the polymer extension limit $L$ has no effect on the short vertical chain formation of particles. However our simulations show that before reaching their vertical chain configurations, the dynamical behavior of the particles and their interactions, differ significantly as the relaxation time $\lambda_{1}$ varies.

\subsection{Settling of 6 and 10 disks}

In order to investigate the influence of the polymer extension limit $L$ on the formation of long chains of particles, we first consider in this section the case of six identical disks, of diameter $d=0.25$, settling in a channel filled with either an Oldroyd-B or a FENE-CR fluid, both with $\eta_{1}=0.26$. The channel is infinitely 


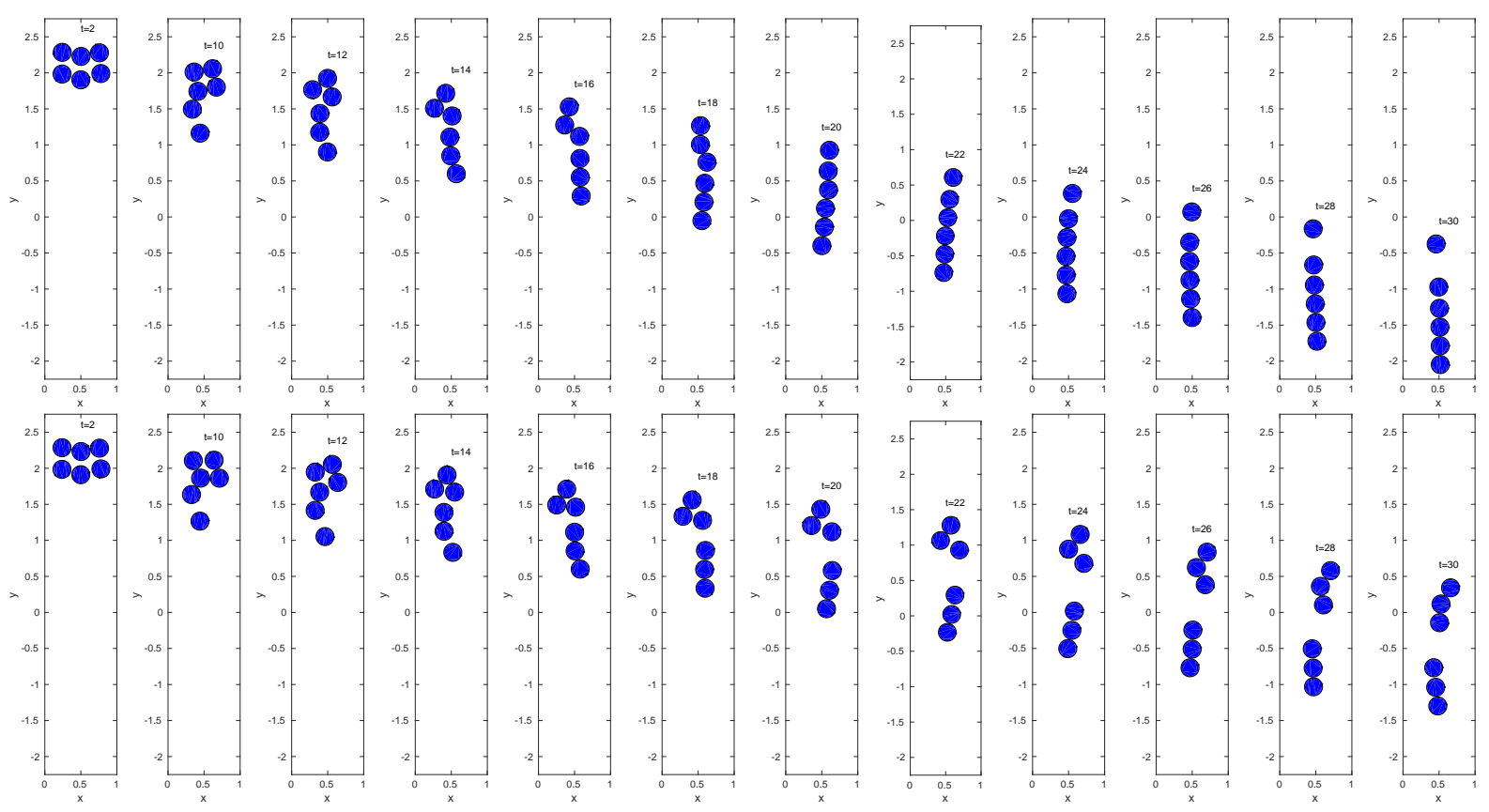

Figure 11: Snapshots of the positions of six disks at $t=2,10,12,14,16,18,20$, ,22, 24, 26, 28, and 30 for $\lambda_{1}=1.3$ in an Oldroyd-B fluid (top) and a FENE-CR fluid with $L=5$ (bottom).

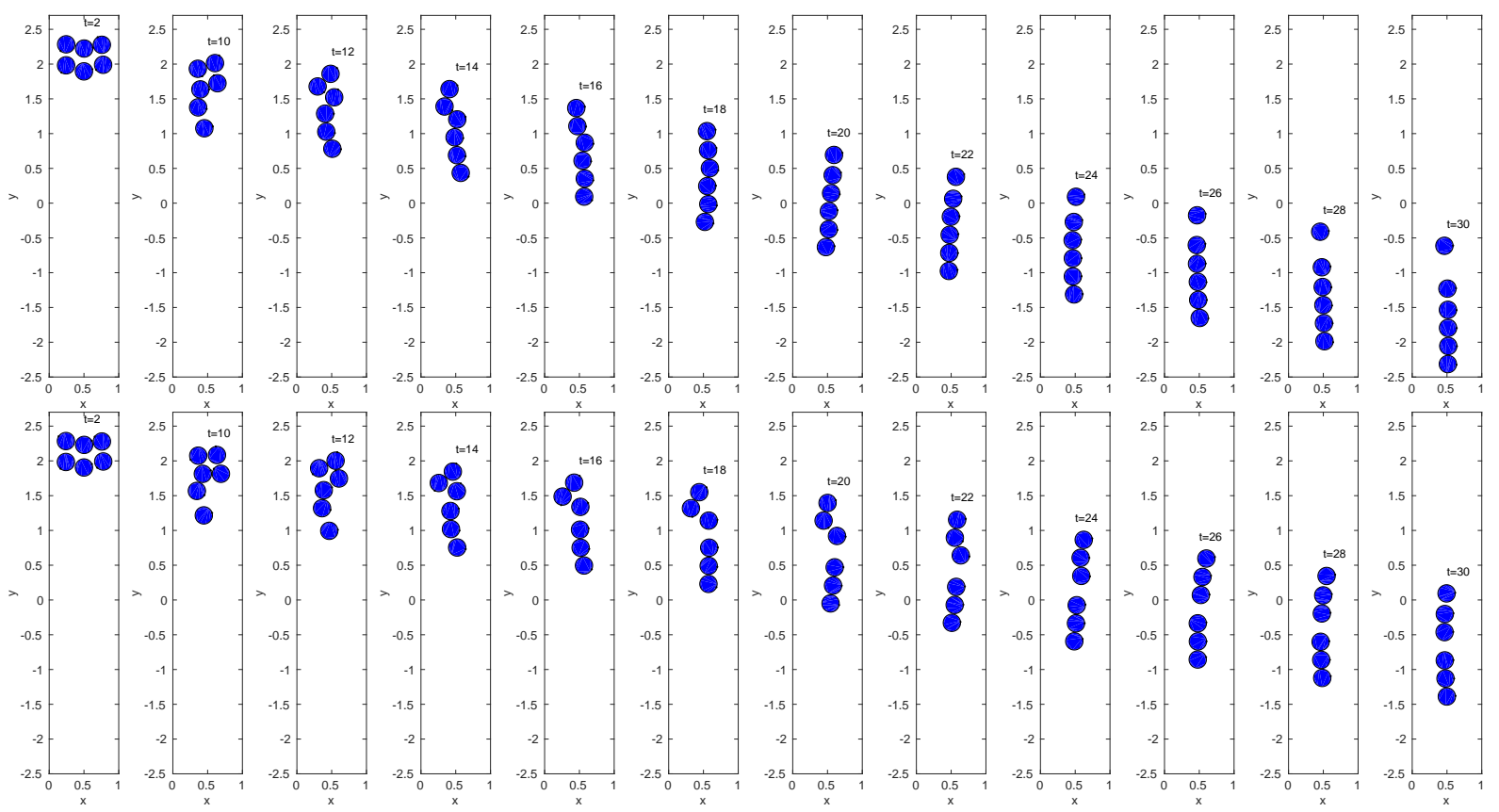

Figure 12: Snapshots of the positions of six disks at $t=2,10,12,14,16,18,20,22,24,26,28$, and 30 for $\lambda_{1}=1.5$ in an Oldroyd-B fluid (top) and a FENE-CR fluid with $L=5$ (bottom). The associated numbers are $\mathrm{Re}=0.149, \mathrm{M}=0.3721, \mathrm{De}=0.9295, \mathrm{E}=6.24$ and $\mathrm{Re}=0.1278, \mathrm{M}=0.3194, \mathrm{De}=0.7978, \mathrm{E}=6.24$ for the Oldroyd-B fluid and the FENE-CR with $L=5$, respectively. 


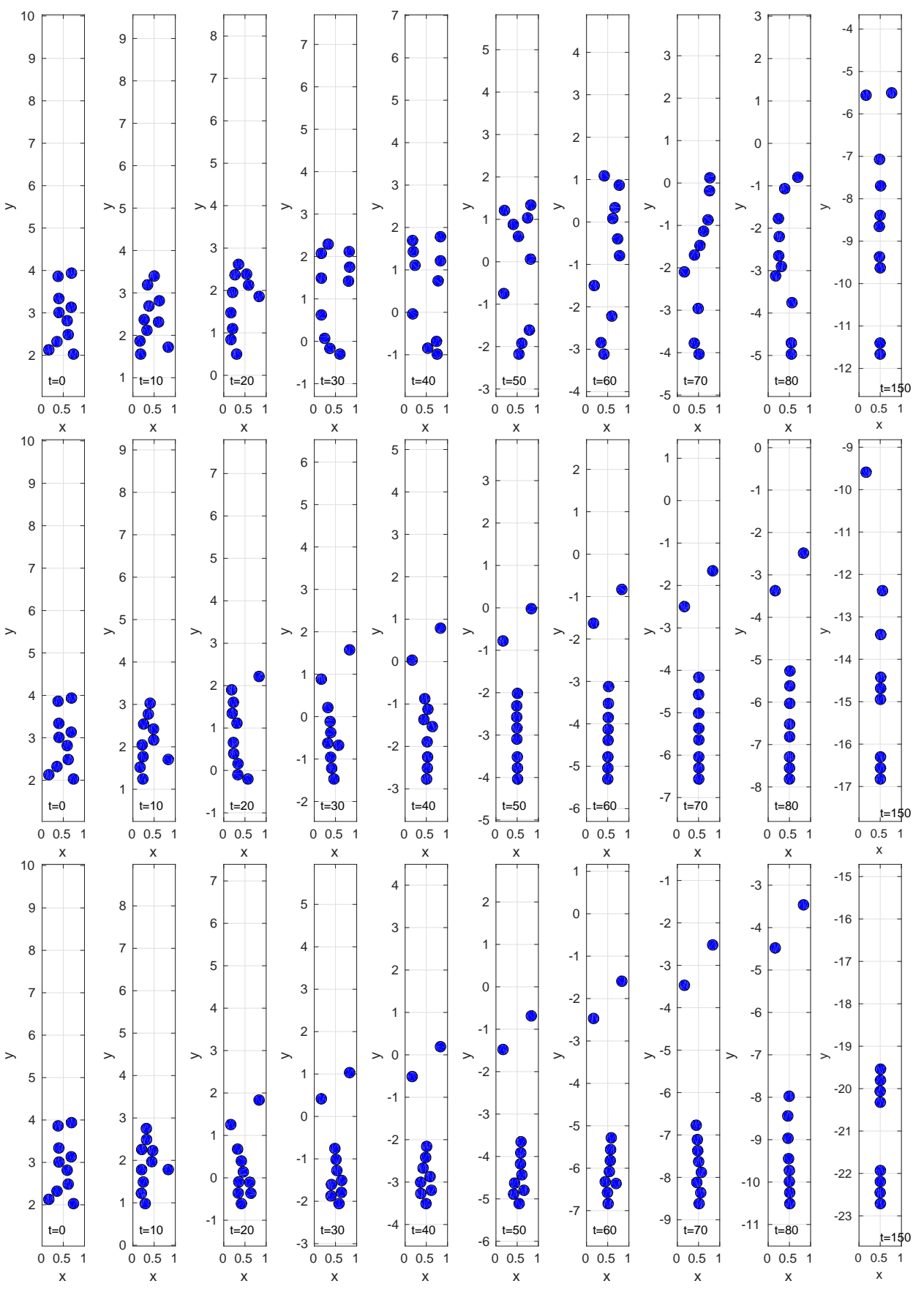

Figure 13: Snapshots of the positions of ten disks at $t=0,10,20,30,40,50,60,70,80$ and 150 in FENECR fluid for $L=2$ (top) and $L=5$ (middle) and in Oldroyd-B fluid (bottom). The associated numbers are $\mathrm{Re}=0.1539, \mathrm{M}=0.4439, \mathrm{De}=1.2804, \mathrm{E}=8.32$ for the Oldroyd-B fluid; $\mathrm{Re}=0.09626, \mathrm{M}=0.2777, \mathrm{De}=0.8009$, $\mathrm{E}=8.32$ for the FENE-CR fluid with $L=2$, and $\mathrm{Re}=0.1092, \mathrm{M}=0.3150, \mathrm{De}=0.9085, \mathrm{E}=8.32$ for the FENECR fluid $L=5$. 
long and has a width of 1 . The computational domain is $\Omega=(0,1) \times(0,7)$ initially and then it moves down with the mass center of the lowest of the six particles. The initial positions of the disks are $(0.23,2.0)$, $(0.5,2.0),(0.78,2.0),(0.22,2.30),(0.5,2.3)$, and $(0.77,2.3)$. The disk density is $\rho_{s}=1.01$ and the fluid density is $\rho_{f}=1$. The relaxation and retardation times are $\lambda_{1}=1.3$ and $\lambda_{2}=\lambda_{1} / 8$, respectively. The mesh sizes for the velocity field, conformation tensor and pressure are $h=1 / 96,1 / 96$, and $1 / 48$, respectively, the time step being 0.0004. The Oldroyd-B related simulations with six disks show that for $t$ sufficiently large the particles line up along the flow direction (the vertical one here), agreeing thus with known observations and experiments. Fig. 11 gives the snapshots at various moments of time of the particles lining up phenomenon. We can see that, after drafting, kissing and chaining, the six particles form approximately a straight line at $t=20$. Then a chain of 5 disks is maintained from $t=22$ to 28 , and during the same time interval the trailing particle separates from the leading five ones. This observation agrees with experiments showing that, sometimes, the last particle in the chain gets detached from the others as discussed in [30]. It is known that long chains fall faster than single particles in fluid. The average terminal velocity is 0.1535 for $26 \leq t \leq 30$, while the Reynolds number is $R e=0.1476$, the Deborah number is $D e=0.7981$, the elasticity number is $\mathrm{E}=5.408$ and the Mach number is $\mathrm{M}=0.3432$. For the FENE-CR model with the polymer extension limit $L=5$, since the viscoelastic fluid has a shorter polymer extension limit, it can not hold all the six disks together as shown in Fig. 11 for $t \geq 16$; instead two chains of three disks are formed and maintained. For this case, the average terminal velocity is 0.1317 for $26 \leq t \leq 30$, while the associated numbers are $\mathrm{Re}=0.1266$, $\mathrm{De}=0.6847, \mathrm{E}=5.408$, and $\mathrm{M}=0.2944$. The particle velocities and trajectories visualized in Fig. 10 for different values of $L$ and $\lambda_{1}=1.3$ show that, if $L$ is about 25, the dynamics of the six disks in FENE-CR fluid is almost identical to the one for the Oldroyd-B fluid. For $\lambda_{1}=1.5$, the particle dynamics and chain formation are similar to those for $\lambda_{1}=1.3$; however the chains of disks in Fig. 12 straighten out faster comparing to those in Fig. 11, a direct consequence of the stronger normal stress due to the larger value of $\mathrm{E}$.

In the second case considered this section, we have increased the number of disks to ten and have kept all other parameters identical except that the computational domain is $\Omega=(0,1) \times(0,16)$, the relaxation time is $\lambda_{1}=2(\mathrm{E}=8.32)$, and the ten disk mass centers are randomly chosen in the region $(0,1) \times(2,4)$, initially. This initial configuration give us some computational results concerning the effect of the polymer extension limit $L$ on the clustering and chaining of particles. Fig. 13 shows snapshots for both Oldroyd-B and FENE-CR viscoelastic fluids. In the Oldroyd-B fluid, the positions of the 10 disks at different instants of time show that the agglomeration of particles can be held initially in a cluster of 8 disks for $20 \leq t \leq 60$; but later the cluster becomes a long chain around $t=70$; however the formation of a long chain can not be kept due to the detachment of the trailing particle as discussed in the previous case for the six disks. The results of the ten particles settling in a FENE-CR for $L=2$ are quite different from those for the above Oldroyd-B fluid. All disks spread out most of the time. The results of the ten particles settling in an FENE-CR for $L=5$ are different from those for Oldroyd-B fluid since these particles are still relatively easier to break away from the chains and clusters. There are two clusters for $20 \leq t \leq 40$; once the disks in these two clusters all line up, the last disk in the chain of more than 3 disks keep breaking away. Finally particles regroup and two chains of three disks are formed for the case of $L=5$. In Fig. 13, there are chains of two disks and three disk in FENE-CR fluids for $L=2$ and 5 , respectively, and chains of four disks in the Oldroyd-B fluid at $t=150$. The numerical results for three cases suggest that for smaller values of $L$, the length of the vertical chains is shorter and the size of clusters is smaller.

\section{Conclusion}

In this article, we presented a method for the numerical simulation of circular particles settling in twodimensional channels fully filled with viscoelastic fluids of the Oldroyd-B and FENE-CR types. As in [11] for Oldroyd-B fluids, the computational methodology we employ relies on operator-splitting, a distributed Lagrange multiplier based fictitious domain method, finite element approximations, and a Cholesky factor- 
ization of the conformation tensor à la Lozinski-Owens. The numerical results concerning vertical chain formation suggest that the polymer extension limit coefficient $L$ of the FENE-CR model has no effect in the case of two or three disks settling in a two-dimensional narrow channel (at least for the values of $L$ considered here). On the other hand, for small values of $L$, our results show that the transient state and particle interactions taking place before reaching chain configurations vary significantly as the relaxation time varies. For six disks settling in a FENE-CR viscoelastic fluid, the final chain configuration depends of $L$ : indeed, for $L$ small enough two chains are formed, while the corresponding Oldroyd-B fluid (or a FENE-CR one with $L$ sufficiently large) produces a five particle chain, the sixth (top) particle being detached from the other ones (see Fig. 12). Similar results hold for ten disks (see Fig. 13), and more, showing that, for small values of $L$, FENE-CR fluids can sustain only short particle chains and clusters.

A next step (already in progress) would be to generalize the computational methodology discussed in this article, in order to investigate the settling of spherical balls in three-dimensional channels filled with viscoelastic fluids of various types (a particular attention being given to the influence of the elasticity number $\mathrm{E}$ on the length of the chains and the size of the clusters).

\section{Acknowledgments}

We acknowledge the support of NSF (grant DMS-1418308).

\section{References}

[1] R. P. Chhabram, Bubbles, Drops, and Particles in Non-Newtonian Fluids, CRC Press, Boca Raton, FA, 1993.

[2] G. H. McKinley, Steady and transient motion of spherical particles in viscoelastic liquids, D. De Kee and R. P. Chhabra (eds.), Transport Processes in Bubbles, Drops \& Particles, 2nd ed., Taylor \& Francis, New York, NY, 2002, pp. 338-375.

[3] M. J. Economides and K. G. Nolte, Reservoir Simulation, Prentice Hall, Englewood Cliffs, NJ, 1989.

[4] I. Tomac, M. Gutierrez, Micromechanics of proppant agglomeration during settling in hydraulic fractures, J. Petrol. Explor. Prod. Technol. 5 (2015) 417-434.

[5] F. P. T. Baaijens, Mixed finite element methods for viscoelastic flow analysis: A review, J. NonNewtonian Fluid Mech. 79 (1998) 361-385.

[6] R. Keunings, A survey of computational rheology, D. M. Binding et al. (eds.), Proc. 13th Int. Congr. on Rheology, Vol. 1, British Society of Rheology, Glasgow, 2000, pp. 7-14.

[7] R. Fattal, R. Kupferman, Constitutive laws for the matrix-logarithm of the conformation tensor, J. Non-Newtonian Fluid Mech. 123 (2004) 281-285.

[8] R. Fattal, R. Kupferman, Time-dependent simulation of viscoelastic flows at high Weissenberg number using the log-conformation representation, J. Non-Newtonian Fluid Mech. 126 (2005) 23-37.

[9] Y.-L. Lee, J. Xu, New formulations, positivity preserving discretizations and stability analysis for nonNewtonian flow models, Comput. Methods Appl. Mech. Eng. 195 (2006) 1180-1206.

[10] A. Lozinski, R.G. Owens, An energy estimate for the Oldroyd-B model: theory and applications, J. Non-Newtonian Fluid Mech. 112 (2003) 161-176. 
[11] J. Hao, T.-W. Pan, R. Glowinski, D. D. Joseph, A fictitious domain/distributed Lagrange multiplier method for the particulate flow of Oldroyd-B fluids: A positive definiteness preserving approach, J. Non-Newtonian Fluid Mech. 156 (2009) 95-111.

[12] J. M. Rallison, E. J. Hinch, Do we understand the physics in the constitutive equation?, J. NonNewtonian Fluid Mech. 29 (1988) 37-55.

[13] D. D. Joseph, Fluid Dynamics of Viscoelastic Liquids, Springer, New York, NY, 1990.

[14] M. D. Chilcott and J. M. Rallison, Creeping flow of dilute polymer solutions past cylinders and spheres, J. Non-Newtonian Fluid Mech. 29 (1988) 381-432.

[15] R. Glowinski, T.-W. Pan, T. I. Hesla, D. D. Joseph, J. Périaux, A fictitious domain approach to the direct numerical simulation of incompressible viscous fluid flow past moving rigid bodies: application to particulate flow, J. Comput. Phys. 169 (2001) 363-426.

[16] P. Singh, D. D. Joseph, T. I. Helsa, R. Glowinski, T.-W. Pan, A distributed Lagrange multiplier/fictitious domain method for viscoelastic particulate flows, J. Non-Newtonian Fluid Mech. 91 (2000) 165-188.

[17] Z. Yu, N. Phan-Thien, Y. Fan, R. I. Tanner, Viscoelastic mobility problem of a system of particles, J. Non-Newtonian Fluid Mech. 104 (2002) 87-124.

[18] A. J. Chorin, T. J. R. Hughes, M. F. McCracken, J. E. Marsden, Product formulas and numerical algorithms, Comm. Pure Appl. Math. 31 (1978) 205-256.

[19] R. Glowinski, Finite element methods for incompressible viscous flows, P.G. Ciarlet, J.L. Lions (eds.), Handbook of Numerical Analysis, vol. IX, North-Holland, Amsterdam, 2003, pp. 3-1176.

[20] R. Glowinski, Variational Methods for the Numerical Solution of Nonlinear Elliptic Problem, SIAM, Philadelphia, 2015.

[21] R. Glowinski, S. Osher, W. Jin (eds.), Splitting Methods in Communication and Imaging, Science and Engineering, Springer, 2016.

[22] E. J. Dean, R. Glowinski, A wave equation approach to the numerical solution of the Navier-Stokes equations for incompressible viscous flow, C.R. Acad. Sci. Paris, Série I, t. 325 (1997) 783-791.

[23] P. Singh, L.G. Leal, Finite-Element simulation of the start-up problem for a viscoelastic fluid in an eccentric rotating cylinder geometry using a third-order upwind scheme, Theor. Comput. Fluid Dyn. 5 (1993) 107-137.

[24] H.H. Hu, D. D. Joseph, M. J. Crochet, Direct simulation of fluid particle motions, Theoret. Comput. Fluid Dynamics 3 (1992) 285-306.

[25] T.-W. Pan, R. Glowinski, G. P. Galdi, Direct simulation of the motion of a settling ellipsoid in Newtonian fluid, J. Comput. Applied Math. 149 (2002) 71-82.

[26] J. Feng, P. Y. Huang, D. D. Joseph, Dynamicc simulation of sedimentation of solid particles in an Oldroyd-B fluid, J. Non-Newtonian Fluid Mech. 63 (1996) 63-88.

[27] A. F. Fortes, D. D. Joseph, T. S. Lundgren, Nonlinear mechanics of fluidization of beds of spherical particles, J. Fluid Mech. 177 (1987) 467-483.

[28] P. Y. Huang, H. H. Hu, D. D. Joseph, Direct simulation of the sedimentation of elliptic particles in Oldroyd-B fluids, J. Fluid Mech. 362 (1998) 297-325. 
[29] Y. L. Liu, D. D. Joseph, Sedimentation of particles in polymer solutions, J. Fluid Mech. 255 (1993) 565-595.

[30] N. A. Patankar, H. H. Hu, A numerical investigation of the detachment of the trailing particle from a chain sedimenting in Newtonian and viscoelastic fluids, J. Fluids. Engineering 122 (2000) 517-521. 